\title{
Interpretation of GPR (Ground Penetrating Radar) and soil profiles at selected veredas of the Formoso River Basin, Buritizeiro, Minas Gerais, Brazil
}

Paulo Roberto Antunes Aranha Adolf Heinrich Horn ${ }^{2}$

Paulo Freitas Morais ${ }^{3}$

${ }^{1}$ Department of Geology Instituto de Geociências Universidade Federal de Minas Gerais Av. Antônio Carlos, Belo Horizonte MG Brazil CEP 31207-901

Centro de Pesquisas Professor Manoel Teixeira da Costa Instituto de Geociências

Universidade Federal de Minas Gerais Av. Antônio Carlos, 6627 Belo Horizonte MG Brazil CEP 31207-901

${ }^{3}$ Centro Federal de Educação Tecnológica Celso Suckow da Fonseca Av. Maracanã, 229, Maracanã Rio de Janeiro RJ Brazil CEP 20271-110

Corresponding author aranha1941@gmail.com

\begin{abstract}
RESUMO
Este trabalho apresenta correlação entre os dados do GPR (Ground Penetrating Radar) e a distribuição geoquímica dos perfis de solos de veredas selecionados na bacia do rio Formoso, para mostrar a correlação entre a distribuição vertical das unidades do solo e o perfil do GPR. Essa interpretação é importante para demonstrar de forma fácil a evolução dessas importantes unidades geomorfológicas com o aumento das atividades humanas no cerrado. Assim, é extremamente importante a implementação de mecanismos necessários para a proteção desses ambientes como fontes de água na região. Esta pesquisa pode contribuir para a criação de áreas protegidas de modalidade de uso sustentável, especificamente áreas de interesse ecológico (ARIE) para a proteção das veredas consideradas estratégicas para a conservação do solo e da água.
\end{abstract}

Palavras chave: GPR; Vereda; proteção ambiental; sustentabilidade

\section{ABSTRACT}

This work shows a correlation between the GPR (Ground Penetrating Radar) data and the geochemical distribution of the selected soil profiles in the Formoso river basin, to show the correlation between the vertical distribution of the soil units and the GPR profile. This interpretation is important to easily demonstrate the evolution of these important geomorphological units with the increase in human activities in the cerrado. Thus, it is extremely important to implement the necessary mechanisms to protect these environments as water sources in the region. This research can contribute to the creation of protected areas of sustainable use, specifically areas of ecological interest (ARIE) for the protection of paths considered strategic for the conservation of soil and water.

Keywords: GPR; Vereda; environmental protection; sustainability

\section{INTRODUCTION}

\subsection{LOCALIZATION AND ACCESS}

The studied area, the Formoso River basin, is located in the Buritizeiro County in the northern part of Minas Gerais State - Brazil, specially the southwest portion of the municipality of Buritizeiro (Figure 1) and is limited by the coordinates $15^{\circ} 05^{\prime}$ and $15^{\circ} 36^{\prime}$ latitude south and $44^{\circ} 56^{\prime}$ and $45^{\circ} 26^{\prime}$ longitude west, draining an area of approximately $826 \mathrm{~km}^{2}$. The town of Buritizeiro is approximately $377 \mathrm{~km}$ far from Belo Horizonte, and the county occupies a land area of $7,226 \mathrm{~km}^{2}$. Access to the town is given by federal highway system using BR040 and BR-365 and by State road systems on MG-161, MG-408 and MG-496. 


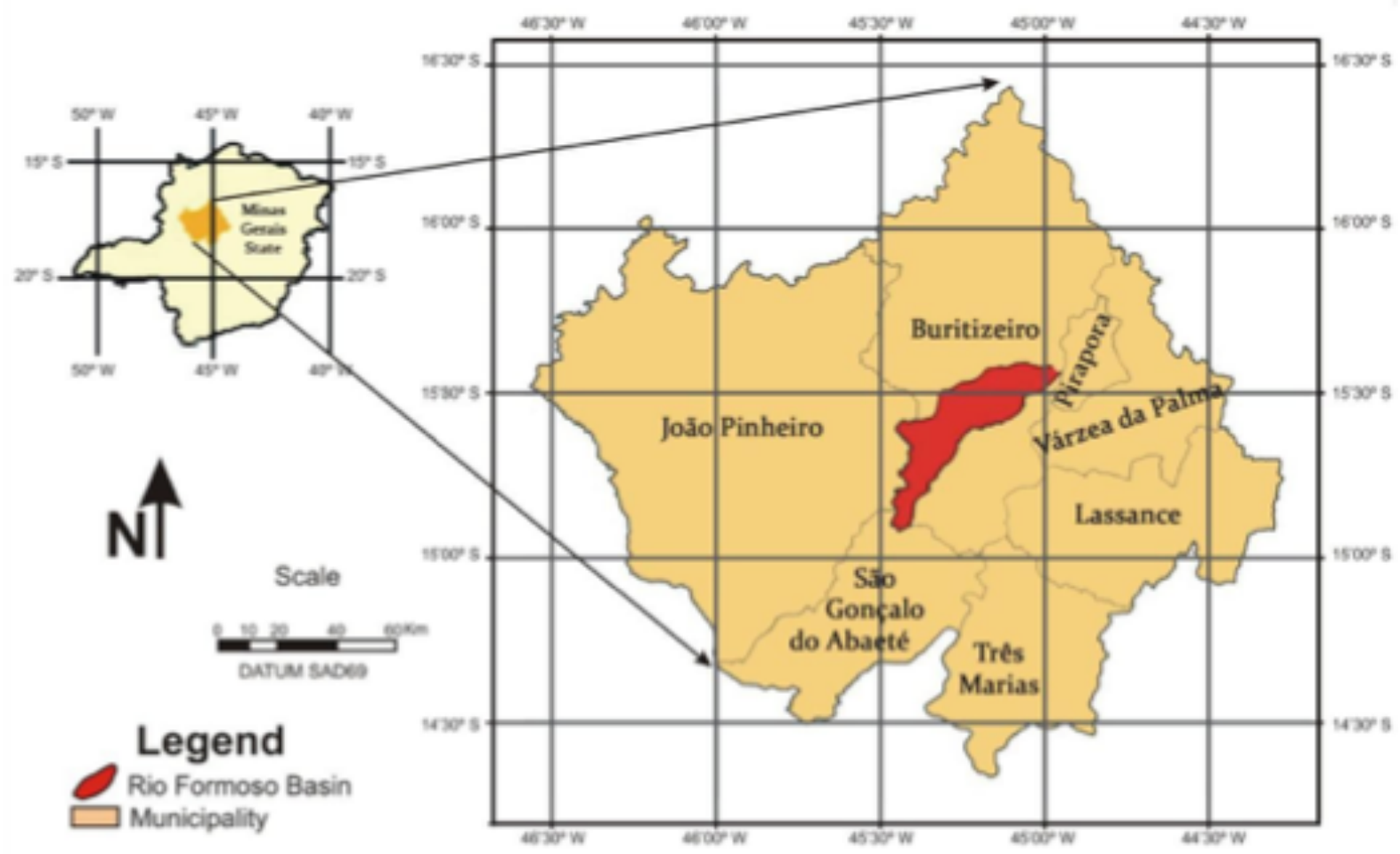

Figure 1

Localization map of the investigated basin. The Formoso river basin is situated at the Buritizeiro county in the State of Minas Gerais (Source: Viana, 2006)

\subsection{VEREDA}

Vereda is an ecosystem that is formed under well-defined moisture conditions and limited to the region of the Cerrado (Savannah), forming normally springs or spring galleries, which supply the major drainage basins of Brazil (VIANA, 2006). The Formoso River basin is an important tributary of the São Francisco River, and is located in the north of the State of Minas Gerais. It hosts innumerous different Vereda types (Figures 2 and 3).

Vereda is also considered a natural ecological corridor in the Cerrado area (MELO, 1992) because the alignments of its buritis palms serve as trail for animals to move, find food and breeding places. According to the same author these areas not only serve as ecological corridors but also have an important function in water distribution to the river, such as retaining water in wet periods and liberating it in dry ones maintaining perennity (continuity) of creeks and rivers. The aquifer exudes, forming wetlands drenches very close to the surface, while the top of the steep-sided sandstone plateaus, works as a recharge area for the aquifers. Another important aspect of veredas, in relation to local communities, is their economic importance due to the large potential of the buriti palm in supplying the various products to local communities, like oil, charcoal, leaves, and construction material.

The soil (substrate) of the veredas is permanently saturated with water, forming in this way islands in arid regions. Due to this, agricultural activities are attracted and concentrated in this part of the Cerrado, influencing the highly sensible equilibrium in this biome. These activities may also change the quality of water and soil and, therefore, influences the whole water support and quality of the connected basins.

The veredas were classified regarding their environmental, geological and morphological features, and three representative sites were selected. The aim of this work was to characterize the soil of the three selected veredas and to determine the changes due to human activities. 


\section{GEOLOGICAL SITUATION}

The Formoso River basin is located in the southern portion of the Sanfranciscana Basin, within the limits of the São Francisco Craton, in the eastern part of the Cretaceous area covering the Minas Gerais State. The rocks that determine the studied area belong to the geological units of Areado e Mata da Corda groups and their corresponding formations (Figure 2).

The Três Marias Formation, the upper unit of Bambuí Group, located in the northern portion of the basin, is represented by their main lithofacies, siltstones with thin sand beds, clay inter-laminations, siltstones with coarsegrained lenses, violet siltstones showing load and drying cracks, sandstones with sigmoidal cross-stratification, sandstones with crossstratification formed by waves, sandstones with hummocky and sandstone with horizontal stratification together with arkosean sandstones and arkoses (CHIAVEGATTO, 1992).

The Areado Group located in the central part of the basin, is represented by the Abaeté and Três Barras formations. The
Abaeté formation represents the basal lithostratigraphic unit of the Sanfranciscana Basin and is represented, in some places of the Formoso River basin, by fluvial conglomerates containing wind canter, gravel and a large volumes of rudicious sediments, deposited under a high energetic regime under arid and semi-arid conditions (SGARBI, 2001). The Três Barras formation is composed of fine to medium sandstones from deposition in dry windy environments and fluvialdeltaic systems, cemented by limestone. The generally pink colored sediments are cut by irregular whitish spots due to selective reduction effects (SGARBI, 1991).

The Mata da Corda Group is composed of volcanic and volcanoclastic rocks covering discordantly the terrigenous Areado Group and is divided into the Patos and Capacete formations. The later one is exposed in the investigated area as vast plateaus (SGARBI, 2001). This plateaus are covered by more recent deposits, mostly elluvial to colluvial and alluvial.

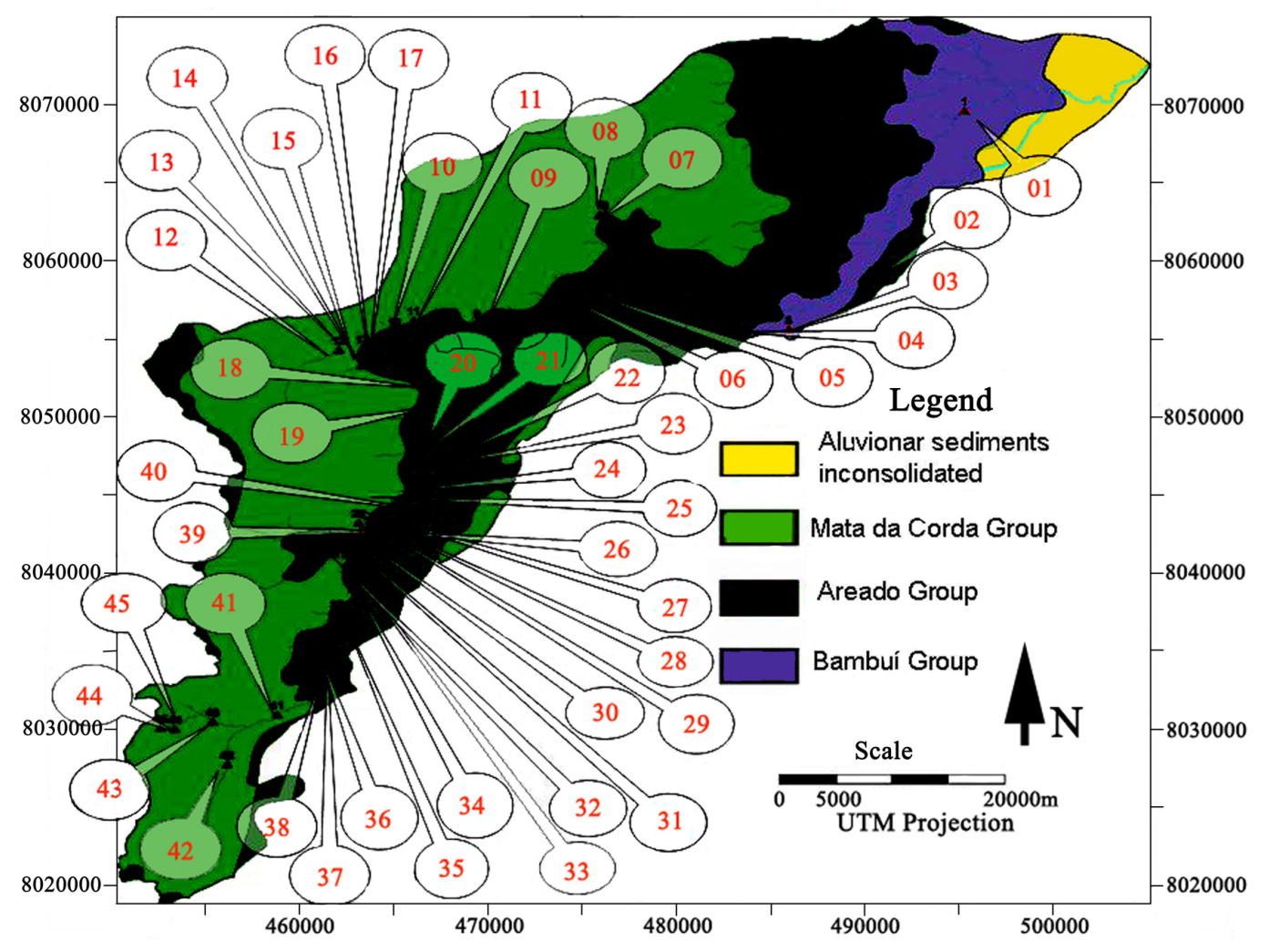

Figure 2

Geological sketch map of the Formoso Basin. The map shows the distribution of the geological units in the investigated basin and the localization of all the veredas. (BAGGIO, 2008). Selected veredas: 42: Vereda Laçador; 09: Vereda Jaraguá; 01: Vereda

Urbana. 


\subsection{PEDOLOGY}

With respect to soil evolution and occurrence in the studied area, it can be shown that in the medium segment has formed mainly Gleisoils with humic-alic affinity, usually associated with the Vereda type in this section (BAGGIO, 2008). Indiscriminate hydromorphic soils (Gleissoils and Organosoils), typically for Vereda formation, are occurring extended in the surrounding and underlying latosoils and quartz rich sands and micro conglomerates, showing the typical dark grey to black colors of Gleis (MELO, 1992).

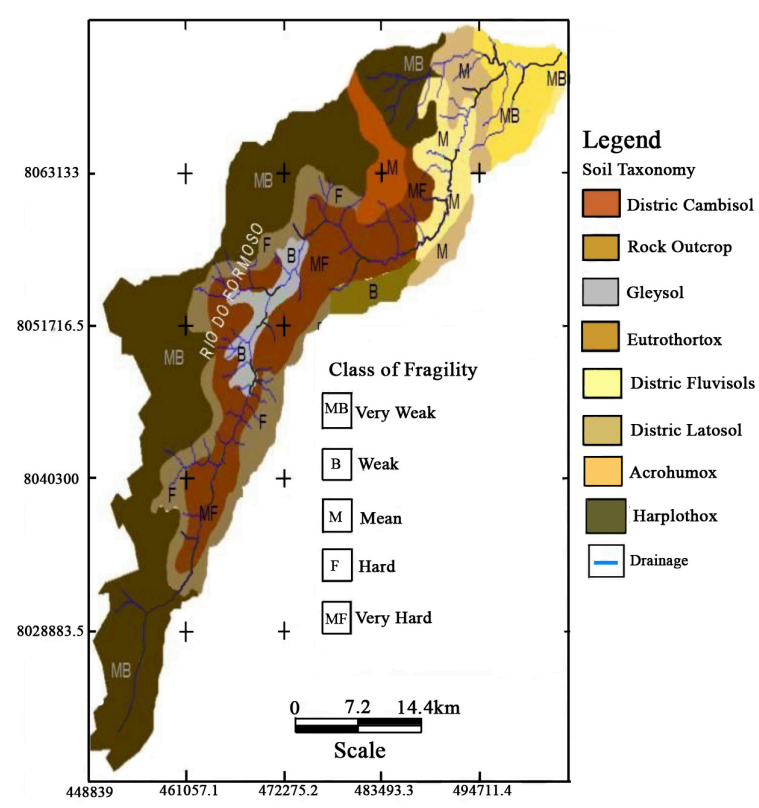

These soils are imperfectly to poorly drained, very poor chemically, strongly acidic ( $\mathrm{pH}$ between 4 and 5.4) with very low base saturation values and a high saturation in aluminum. The gleisoils possess profile with an $\mathrm{ACg}$ horizons sequence, in which the $\mathrm{A}$ appears much darkened by organic matter, and $\mathrm{C}$ sometimes can be subdivided into $\mathrm{C} 1 \mathrm{~g}$ and C2g (MELO, 1992).

A distribution of the soils and the soil use in the investigated area can be seen in Figure 3. Figure 4 shows the surface soil color distribution of the investigated veredas.

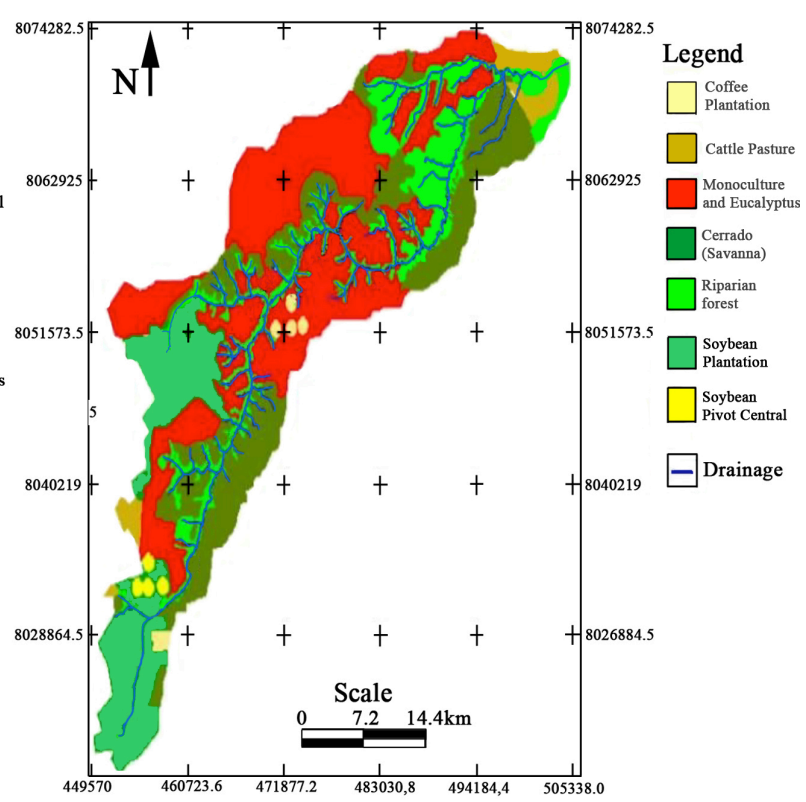

Figure 3

Distribuition of soils and the land use (agriculture; cattle, forests) in the basin of Formoso river (Adapted from Baggio, 2008)
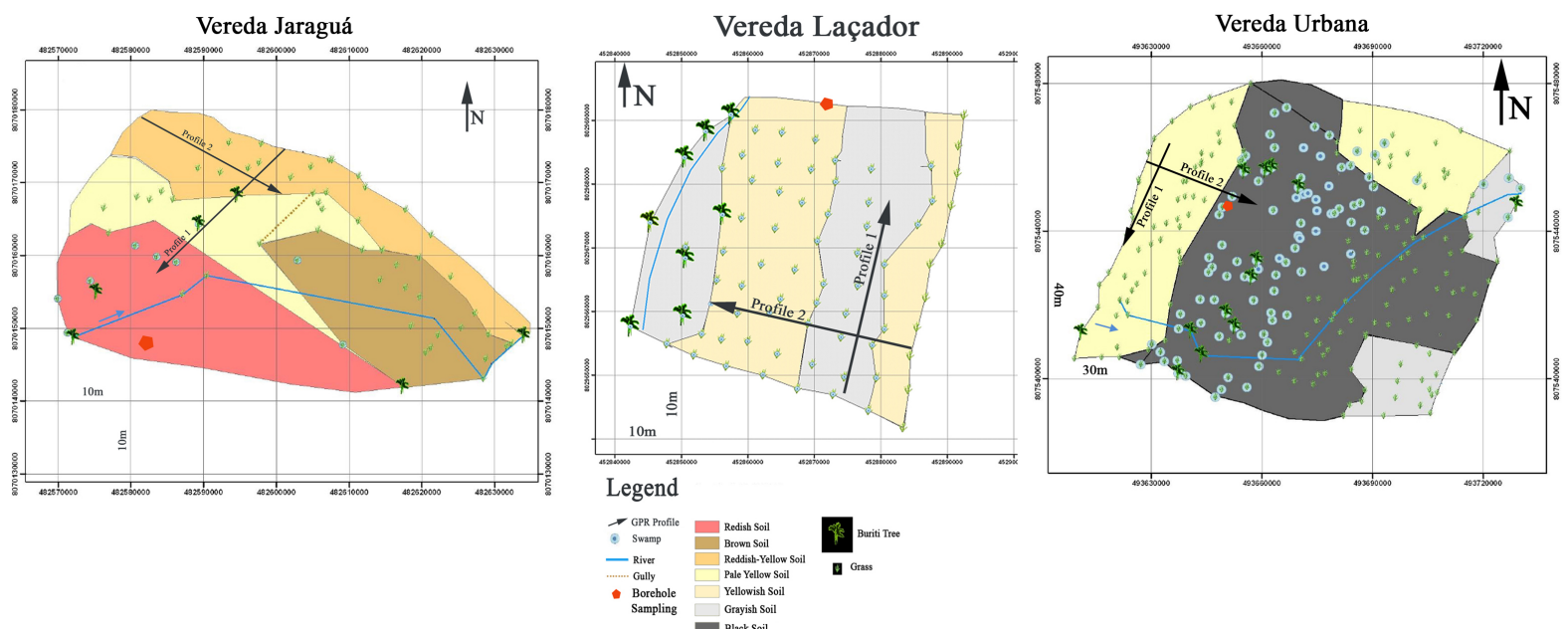

Figure 4

Location and soil maps of the three investigated Veredas, the GPR profiles and the sampled drill holes.

\section{METHODOLOGY}




\subsection{GPR INVESTIGATIONS}

The geophysical data were obtained in the study area using a GPR, RAMAC equipment, from Mala GeoScience. Concomitantly, CMP (common mid point) profiles were done to define the velocity profile of the EM wave in the investigated area. Antennas with a central frequency of 100 $\mathrm{MHz}$ were used. The survey was performed with constant distance antennas (common offset) and the system was transported along a direction to obtain a profile of reflections versus position. The 16 stack mode was applied in order to increase the signal/noise ratio of the emitted wave, improving the

\subsection{SOIL SAMPLING}

Soil samples were collected in the selected veredas in horizontal and vertical profiles. In horizontal profiles one sample (5 $\mathrm{kg}$ ) was taken every $2 \mathrm{~m}$ in the upper $20 \mathrm{~cm}$ of the soil. The soil sampling was conducted in December 2008, February and May 2009, based on the USP-EPA $(1992 ; 2007)$ methodology of the soil sampling (Figure 4). On selected positions, vertical sampling was carried out taking $2-3 \mathrm{~kg}$ samples every $20 \mathrm{~cm}$ down to a maximum quality of data acquired in the field. The other parameters used for the elaboration of the profiles were sampling frequency approximately 10 times of the central frequency of used antenna and a $450 \mathrm{~ms}$ time window. The spacing between the data acquisition points was $0,10 \mathrm{~m}$.

The data processing aimed to improve the quality of the results of the field data, so that the interpretation of the images presented a better accuracy. The edition comprised the organization of data, declipping, dewow, set time zero, migration, filtering, elevation statics, and depth conversion.

depth of $2 \mathrm{~m}$ (Figure 5). The samples were obtained by "Wolf mouth" type samplers and for deeper profiles a normal soil sampler. At the Laçador Vereda soil samples were obtained along six stratigraphic profiles. In Jaraguá Vereda, samples were collected along two stratigraphic profiles. In the Urbano Vereda, the samples were taken along two trenches opened by caving.
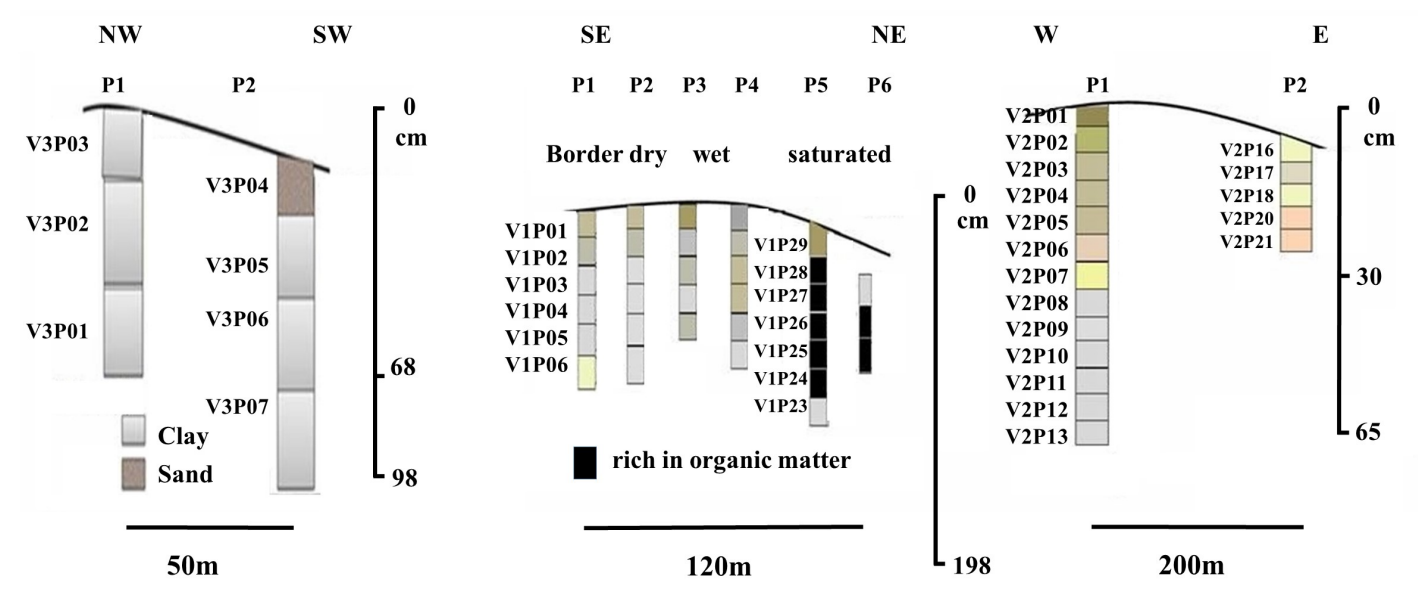

Figure 5

Indication of profiles and soils of the three investigated veredas. Left: Urbano; Middle: Laçador; right: Jaraguá.

\subsection{LABORATORY TREATMENT}

After collection, samples were homogenized, packed in plastic bags and transported to the environmental laboratory (NGqA) of the Manoel Teixeira da Costa Research Center (CPMTC/IGC/UFMG) where preparation and chemical analyses were carried out.

The samples were placed in paper trays and dried at room temperature for a period of 15 to 20 days. After drying, $500 \mathrm{~g}$ of each soil sample were submitted to particle size separation following the technical standard ABNT NBR 7181/1982 (ABNT, 1984).

The wet and dry soil colors were determined using Soil Color Charts (MUNSELL, 1975) as well as the soil $\mathrm{pH}$ in water (Table 1) and the cationic exchange capacity (CTC), using Embrapa procedure (EMPRAPA, 1997; 
Table 2), the organic matter content was determined by colorimetric method and the metals by ICP-OES (Tables 3a, 3b 3c).
Qualitative to semi quantitative mineralogical analysis was performed in the Laboratory of CPMTC by X-ray Diffraction.

Table 1: $\mathrm{pH}$ obtained in the profiles. V1: Vereda Laçador; V2: Vereda Jaraguá; V3: Vereda Urbana. Numbers from figure 5.

\begin{tabular}{|c|c|c|c|c|c|c|c|c|}
\hline Sample & Depth $(\mathrm{cm})$ & $\mathrm{pH}$ & Sample & Depth $(\mathrm{cm})$ & $\mathrm{pH}$ & Sample & Depth $(\mathrm{cm})$ & $\mathrm{pH}$ \\
\hline V1S01 & $0-20$ & 6,51 & V2S01 & $0-20$ & 4,57 & V3P01 & $39-52$ & 2,59 \\
\hline V1S02 & $0-20$ & 6,16 & $\mathrm{~V} 2 \mathrm{~S} 02$ & $0-20$ & 4,50 & V3P02 & $29-35$ & 1,68 \\
\hline V1S03 & $0-20$ & 4,36 & $\mathrm{~V} 2 \mathrm{~S} 03$ & $0-20$ & 4,38 & V3P03 & $09-23$ & 2,4 \\
\hline V1S05 & $0-20$ & 6,59 & $\mathrm{~V} 2 \mathrm{~S} 04$ & $0-20$ & 3,08 & V3P04 & $0-20$ & 3,28 \\
\hline V1S06 & $0-20$ & 4,72 & $\mathrm{~V} 2 \mathrm{~S} 05$ & $0-20$ & 4,70 & V3P05 & $20-40$ & 5,15 \\
\hline V1S07 & $0-20$ & 5,81 & V2S06 & $0-20$ & 4,46 & V3P06 & $48-65$ & 4 \\
\hline V1S08 & $0-20$ & 4,41 & V2S07 & $0-20$ & 3,99 & V3P07 & $76-96$ & 5,34 \\
\hline V1S08B & $0-20$ & 6,07 & V2S08 & $0-20$ & 5,23 & V3S01 & $0-20$ & 4,76 \\
\hline V1S09 & $0-20$ & 6,52 & V2S09 & $0-20$ & 5,38 & V3S02 & $0-20$ & 4,8 \\
\hline V1S10 & $0-20$ & 4,68 & $\mathrm{~V} 2 \mathrm{~S} 10$ & $0-20$ & 4,72 & V3S03 & $0-20$ & 4,07 \\
\hline V1S11 & $0-20$ & 4,73 & V2S11 & $0-20$ & 5,78 & V3S04 & $0-20$ & 4,97 \\
\hline V1S12 & $0-20$ & 5,66 & V2S12 & $0-20$ & 5,12 & V3S05 & $0-20$ & 4,71 \\
\hline V1S13 & $0-20$ & 4,57 & V2S13 & $0-20$ & 4,73 & V3S06 & $0-20$ & 5,73 \\
\hline V1S14 & $0-20$ & 5,38 & V2S14 & $0-20$ & 4,89 & V3S07 & $0-20$ & 2,65 \\
\hline V1S15 & $0-20$ & 5,27 & V2S15 & $0-20$ & 5,42 & V3S08 & $0-20$ & 1,22 \\
\hline V1S16 & $0-20$ & 5,06 & V2S16 & $0-20$ & 4,17 & V3S09 & $0-20$ & 3,1 \\
\hline V1S17 & $0-20$ & 5,18 & V2S18 & $0-20$ & 5,31 & V3S10 & $0-20$ & 2,45 \\
\hline V1S18 & $0-20$ & 4,1 & V2S19 & $0-20$ & 5,31 & V3S11 & $0-20$ & 0,75 \\
\hline V1S19 & $0-20$ & 2,87 & $\mathrm{~V} 2 \mathrm{~S} 22$ & $0-20$ & 6,26 & V3S12 & $0-20$ & 1,88 \\
\hline V1S20 & $0-20$ & 4,54 & $\mathrm{~V} 2 \mathrm{~S} 23$ & $0-20$ & 6,04 & V3S13 & $0-20$ & 1,21 \\
\hline V1S21 & $0-20$ & 5,25 & $\mathrm{~V} 2 \mathrm{~S} 24$ & $0-20$ & 6,03 & V3S14 & $0-20$ & 5,04 \\
\hline V1S22 & $0-20$ & 5,05 & $\mathrm{~V} 2 \mathrm{~S} 25$ & $0-20$ & 3,78 & V3S15 & $0-20$ & 4,53 \\
\hline V1S23 & $0-20$ & 4,98 & V2P01 & $0-5$ & 3,41 & V3S16 & $0-20$ & 4,87 \\
\hline V1S24 & $0-20$ & 4,34 & $\mathrm{~V} 2 \mathrm{P} 02$ & $15-20$ & 5,29 & V3S17 & $0-20$ & 4,48 \\
\hline V1S24A & $0-20$ & 3,99 & V2P03 & $15-20$ & 4,22 & V3S18 & $0-20$ & 5,36 \\
\hline V1S25 & $0-20$ & 4,12 & V2P04 & $15-20$ & 3,08 & V3S19 & $0-20$ & 5,7 \\
\hline V1S26 & $0-20$ & 5,09 & V2P05 & $20-25$ & 4,27 & V3S20 & $0-20$ & 1,53 \\
\hline V1S27 & $0-20$ & 5,08 & V2P06 & $25-30$ & 4,48 & V3S21 & $0-20$ & 3,03 \\
\hline V1S28 & $0-20$ & 3,4 & V2P07 & $30-35$ & 4,31 & V3S22 & $0-20$ & 1,69 \\
\hline V1S29 & $0-20$ & 5,37 & V2P08 & $35-40$ & 5,57 & V3S23 & $0-20$ & 1,69 \\
\hline V1S30 & $0-20$ & 3,71 & V2P09 & $40-45$ & 4,88 & V3S24 & $0-20$ & 3,03 \\
\hline V1S31 & $0-20$ & 4,25 & $\mathrm{~V} 2 \mathrm{P} 10$ & $45-50$ & 4,33 & V3S25 & $0-20$ & 3,06 \\
\hline V1S32 & $0-20$ & 4,81 & V2P11 & $50-55$ & 5,75 & V3S26 & $0-20$ & 4,18 \\
\hline V1P01 & $0-20$ & 6,67 & $\mathrm{~V} 2 \mathrm{P} 12$ & $55-60$ & 4,43 & V3S27 & $0-20$ & 3,83 \\
\hline V1P02 & $20-40$ & 6,49 & V2P13 & $60-65$ & 4,32 & V3S28 & $0-20$ & 3,04 \\
\hline V1P03 & $40-60$ & 6,79 & V2P16 & $0-5$ & 4,08 & V3S29 & $0-20$ & 3,38 \\
\hline V1P04 & $60-80$ & 6,15 & V2P17 & $0-15$ & 4,14 & V3S30 & $0-20$ & 3,14 \\
\hline V1P05 & $80-100$ & 4,5 & V2P18 & $15-20$ & 4,77 & & & \\
\hline V1P06 & $100-127$ & 4,57 & V2P20 & $15-20$ & 5,03 & & & \\
\hline V1P07 & 0-17 & 5,77 & $\mathrm{~V} 2 \mathrm{P} 21$ & $20-25$ & 4,25 & & & \\
\hline V1P08 & $17-34$ & 5,92 & & & & & & \\
\hline V1P09 & $34-51$ & 4,44 & & & & & & \\
\hline V1P10 & $51-68$ & 5,58 & & & & & & \\
\hline V1P11 & $68-85$ & 3,45 & & & & & & \\
\hline V1P12 & $85-103$ & 3,04 & & & & & & \\
\hline V1P13 & $0-20$ & 6,67 & & & & & & \\
\hline V1P14 & $20-40$ & 6,97 & & & & & & \\
\hline V1P15 & $40-60$ & 6,64 & & & & & & \\
\hline V1P16 & $60-80$ & 6,41 & & & & & & \\
\hline V1P17 & $80-100$ & 6,32 & & & & & & \\
\hline V1P18 & 0-19 & 3,48 & & & & & & \\
\hline V1P19 & $19-38$ & 2,79 & & & & & & \\
\hline V1P20 & $38-57$ & 4,03 & & & & & & \\
\hline V1P21 & $57-76$ & 3,87 & & & & & & \\
\hline V1P22 & $76-95$ & 3,88 & & & & & & \\
\hline V1P23 & $95-114$ & 3,18 & & & & & & \\
\hline V1P24 & $160-180$ & 2,49 & & & & & & \\
\hline V1P25 & $128-148$ & 2,47 & & & & & & \\
\hline V1P26 & $100-120$ & 3,13 & & & & & & \\
\hline V1P27 & $70-90$ & 2,44 & & & & & & \\
\hline V1P28 & $40-60$ & 2,52 & & & & & & \\
\hline V1P29 & $10-30$ & 3,42 & & & & & & \\
\hline BS & $180-193$ & 2,65 & & & & & & \\
\hline V1P30 & $30-53$ & 2,75 & & & & & & \\
\hline V1P31 & $53-76$ & 2,02 & & & & & & \\
\hline V1P32 & $76-100$ & 1,41 & & & & & & \\
\hline
\end{tabular}


Table 2 - Values of determined CTC exchange capacity.V1: Vereda Laçador; V2: Vereda Jaraguá; V3: Vereda Urbana. Numbers from figure 5 .

\begin{tabular}{|c|c|c|c|}
\hline Indication & Vereda & Samples used & $T\left(\mathrm{cmol}_{\mathrm{c}} / \mathrm{dm}^{3}\right)$ \\
\hline CTC 01 & V3 & V3P01 + V3P02 + V3P03 & 5,33 \\
\hline CTC 02 & $\mathrm{~V} 3$ & V3P04 & 1,42 \\
\hline CTC 03 & V3 & $\mathrm{V} 3 \mathrm{P} 05+\mathrm{V} 3 \mathrm{P} 06+\mathrm{V} 3 \mathrm{P} 07$ & 4,34 \\
\hline CTC 04 & $\mathrm{~V} 2$ & $\mathrm{~V} 2 \mathrm{P} 01+\mathrm{V} 2 \mathrm{P} 02+\mathrm{V} 2 \mathrm{P} 03+\mathrm{V} 2 \mathrm{P} 04$ & 1,5 \\
\hline CTC 05 & $\mathrm{~V} 2$ & $\mathrm{~V} 2 \mathrm{P} 06+\mathrm{V} 2 \mathrm{P} 07+\mathrm{V} 2 \mathrm{P} 08+\mathrm{V} 2 \mathrm{P} 09$ & 0,79 \\
\hline CTC 06 & $\mathrm{~V} 2$ & $\mathrm{~V} 2 \mathrm{P} 10+\mathrm{V} 2 \mathrm{P} 11+\mathrm{V} 2 \mathrm{P} 12+\mathrm{V} 2 \mathrm{P} 13$ & 1,34 \\
\hline СТC 07 & $\mathrm{~V} 2$ & $\mathrm{~V} 2 \mathrm{P} 16+\mathrm{V} 2 \mathrm{P} 17+\mathrm{V} 2 \mathrm{P} 18+\mathrm{V} 2 \mathrm{P} 21$ & 1,24 \\
\hline CTC 08 & V1 & $\mathrm{V} 1 \mathrm{P} 01+\mathrm{V} 1 \mathrm{P} 02+\mathrm{V} 1 \mathrm{P} 04+\mathrm{V} 1 \mathrm{P} 05$ & 5,24 \\
\hline СТC 09 & V1 & $\mathrm{V} 1 \mathrm{P} 07+\mathrm{V} 1 \mathrm{P} 12$ & 2,04 \\
\hline CTC 10 & V1 & $\mathrm{V} 1 \mathrm{P} 13+\mathrm{V} 1 \mathrm{P} 14+\mathrm{V} 1 \mathrm{P} 15+\mathrm{V} 1 \mathrm{P} 16$ & 5,57 \\
\hline CTC 11 & V1 & $\mathrm{V} 1 \mathrm{P} 18+\mathrm{V} 1 \mathrm{P} 19+\mathrm{V} 1 \mathrm{P} 22+\mathrm{V} 1 \mathrm{P} 23$ & 2,53 \\
\hline CTC 12 & $\mathrm{~V} 1$ & $\mathrm{~V} 1 \mathrm{P} 30+\mathrm{V} 1 \mathrm{P} 31+\mathrm{V} 1 \mathrm{P} 32$ & 3,23 \\
\hline
\end{tabular}

Table 3a - Heavy metal contents $(\mathrm{mg} / \mathrm{kg})$ in soil profile from Vereda Laçador. Depth in $\mathrm{cm}$.

\begin{tabular}{|c|c|c|c|c|c|c|c|c|c|}
\hline Sample & Depth & $\mathrm{Cr}$ & Co & $\mathrm{Cu}$ & Cd & $\mathbf{N i}$ & $\mathbf{Z n}$ & $\mathbf{B a}$ & $\mathbf{P b}$ \\
\hline V2S01 & $0-20$ & 23,7 & 1,6 & 13,5 & 0,81 & 0,34 & 18,9 & 26,3 & 35,4 \\
\hline V2S02 & $0-20$ & 25,8 & 2,1 & 14,3 & 0,84 & 0,34 & 16,4 & 32,7 & 33,2 \\
\hline V2S03 & $0-20$ & 67,1 & 3,4 & 17,0 & 1,26 & 0,34 & 31,3 & 45,8 & 43,4 \\
\hline V2S04 & $0-20$ & 46,0 & 1,8 & 15,5 & 0,85 & 0,34 & 17,4 & 37,8 & 40,8 \\
\hline V2S05 & $0-20$ & 35,8 & 2,9 & 16,8 & 1,03 & 1,31 & 21,7 & 63,5 & 44,5 \\
\hline V2S06 & $0-20$ & 23,0 & 1,6 & 13,0 & 0,81 & 0,34 & 12,5 & 30,4 & 31,0 \\
\hline V2S07 & $0-20$ & 35,6 & 2,8 & 15,8 & 1,04 & 0,34 & 29,0 & 60,9 & 43,2 \\
\hline V2S08 & $0-20$ & 70,0 & 4,1 & 13,9 & 1,01 & 0,34 & 16,3 & 33,8 & 39,0 \\
\hline V2S09 & $0-20$ & 37,0 & 3,6 & 18,3 & 1,16 & 0,87 & 24,9 & 62,7 & 58,3 \\
\hline V2S10 & $0-20$ & 39,6 & 1,3 & 15,6 & 0,73 & 0,34 & 25,3 & 18,0 & 37,6 \\
\hline V2S11 & $0-20$ & 22,7 & 1,1 & 14,4 & 0,70 & 0,34 & 14,4 & 47,1 & 32,6 \\
\hline V2S12 & $0-20$ & 18,6 & 0,9 & 13,6 & 0,61 & 0,34 & 13,1 & 22,7 & 21,8 \\
\hline V2S13 & $0-20$ & 40,4 & 2,4 & 14,3 & 1,12 & 0,34 & 15,0 & 30,4 & 57,6 \\
\hline V2S14 & $0-20$ & 37,3 & 2,4 & 16,1 & 0,84 & 0,34 & 18,3 & 57,1 & 42,2 \\
\hline V2S15 & $0-20$ & 33,7 & 1,3 & 16,9 & 0,76 & 0,34 & 13,9 & 30,1 & 46,0 \\
\hline V2S16 & $0-20$ & 53,3 & 2,5 & 18,1 & 1,05 & 1,07 & 17,9 & 34,1 & 53,2 \\
\hline V2S18 & $0-20$ & 54,3 & 1,6 & 19,0 & 0,93 & 0,34 & 15,5 & 24,2 & 47,9 \\
\hline V2S19 & $0-20$ & 146,0 & 3,4 & 20,0 & 1,29 & 4,63 & 27,2 & 60,6 & 80,0 \\
\hline V2S22 & $0-20$ & 22,2 & 2,0 & 9,8 & 1,01 & 0,34 & 12,0 & 10,8 & 23,8 \\
\hline V2S23 & $0-20$ & 35,9 & 1,5 & 15,7 & 0,77 & 0,34 & 9,0 & 67,3 & 55,4 \\
\hline V2S24 & $0-20$ & 50,5 & 1,4 & 12,4 & 0,77 & 0,34 & 11,8 & 14,5 & 38,7 \\
\hline V2S25 & $0-20$ & 26,2 & 1,5 & 26,1 & 0,95 & 0,34 & 26,4 & 89,8 & 91,7 \\
\hline V2P01 & $0-5$ & 37,8 & 2,8 & 16,3 & 0,89 & 0,34 & 25,7 & 55,1 & 41,9 \\
\hline V2P02 & $5-10$ & 37,2 & 2,6 & 15,4 & 0,92 & 0,34 & 13,2 & 59,2 & 40,0 \\
\hline V2P03 & $10-15$ & 40,5 & 2,9 & 17,2 & 1,28 & 0,34 & 14,6 & 79,9 & 53,7 \\
\hline V2P04 & $15-20$ & 45,9 & 1,9 & 21,2 & 0,92 & 0,34 & 13,5 & 73,7 & 52,9 \\
\hline V2P05 & $20-25$ & 62,3 & 1,9 & 17,1 & 0,85 & 0,34 & 17,1 & 38,3 & 52,2 \\
\hline V2P06 & $25-30$ & 71,2 & 2,0 & 19,1 & 0,79 & 0,67 & 15,7 & 60,8 & 48,9 \\
\hline V2P07 & $30-35$ & 66,6 & 1,2 & 13,9 & 0,64 & 0,34 & 15,6 & 36,8 & 41,8 \\
\hline V2P08 & $35-40$ & 98,3 & 2,4 & 17,4 & 1,03 & 0,34 & 25,4 & 80,9 & 69,3 \\
\hline V2P09 & $40-45$ & 81,2 & 2,0 & 16,1 & 0,90 & 0,34 & 14,9 & 52,9 & 46,9 \\
\hline V2P10 & $45-50$ & 104,1 & 2,2 & 22,7 & 1,16 & 0,34 & 10,5 & 124,5 & 67,4 \\
\hline V2P11 & $50-55$ & 84,9 & 2,3 & 13,4 & 1,07 & 0,34 & 12,1 & 48,8 & 55,0 \\
\hline V2P12 & $55-60$ & 101,8 & 1,3 & 21,7 & 0,73 & 0,34 & 9,9 & 90,8 & 72,0 \\
\hline V2P13 & $60-65$ & 90,3 & 1,7 & 17,6 & 0,85 & 0,34 & 11,4 & 63,7 & 53,3 \\
\hline V2P16 & $0-5$ & 91,6 & 3,0 & 13,3 & 1,21 & 2,99 & 15,8 & 22,2 & 42,2 \\
\hline V2P17 & $5-10$ & 74,1 & 2,4 & 12,3 & 1,07 & 0,34 & 15,3 & 21,8 & 33,9 \\
\hline V2P18 & $10-15$ & 89,8 & 2,4 & 18,0 & 0,99 & 3,49 & 27,9 & 36,6 & 41,9 \\
\hline V2P20 & $15-20$ & 171,3 & 4,0 & 17,2 & 1,61 & 7,34 & 33,4 & 59,4 & 94,4 \\
\hline V2P21 & $20-25$ & 42,1 & 3,3 & 15,4 & 1,14 & 0,34 & 18,9 & 46,1 & 40,8 \\
\hline
\end{tabular}


Table 3b - Heavy metal contents $(\mathrm{mg} / \mathrm{kg})$ in soil profile from Vereda Jaraguá. Depth in $\mathrm{cm}$.

\begin{tabular}{|c|c|c|c|c|c|c|c|c|c|}
\hline Amostra & Depth (cm) & $\mathrm{Cr}$ & Co & $\mathrm{Cu}$ & Cd & $\mathbf{N i}$ & $\mathbf{Z n}$ & Ba & $\mathbf{P b}$ \\
\hline V1S01 & $0-20$ & 136,4 & 1,5 & 28,0 & 1,2 & 1,0 & 40,8 & 34,0 & 120,2 \\
\hline V1S02 & $0-20$ & 121,9 & 1,4 & 22,0 & 0,9 & 0,3 & 18,9 & 5,6 & 100,4 \\
\hline V1S03 & $0-20$ & 145,3 & 1,8 & 22,5 & 1,2 & 0,9 & 21,1 & 9,1 & 117,6 \\
\hline V1S05 & $0-20$ & 138,7 & 1,3 & 21,2 & 1,1 & 0,3 & 38,4 & 15,9 & 123,8 \\
\hline V1S06 & $0-20$ & 140,3 & 1,2 & 22,2 & 0,9 & 0,3 & 18,1 & 10,3 & 119,9 \\
\hline V1S07 & $0-20$ & 147,5 & 1,6 & 21,0 & 1,1 & 0,3 & 21,1 & 6,0 & 108,0 \\
\hline V1S08 & $0-20$ & 157,3 & 1,8 & 32,4 & 1,0 & 0,3 & 21,6 & 11,7 & 125,3 \\
\hline V1S08B & $0-20$ & 142,2 & 1,2 & 25,5 & 0,9 & 0,3 & 28,1 & 9,3 & 119,4 \\
\hline V1S09 & $0-20$ & 145,4 & 1,2 & 28,1 & 1,0 & 0,3 & 28,7 & 14,5 & 121,5 \\
\hline V1S10 & $0-20$ & 163,9 & 1,3 & 30,5 & 1,0 & 0,3 & 21,0 & 9,1 & 134,3 \\
\hline V1S11 & $0-20$ & 157,0 & 1,8 & 25,5 & 1,2 & 0,3 & 15,5 & 9,0 & 129,8 \\
\hline V1S12 & $0-20$ & 164,9 & 1,6 & 27,6 & 1,0 & 0,3 & 16,9 & 9,8 & 139,2 \\
\hline V1S13 & $0-20$ & 166,2 & 2,6 & 22,9 & 1,6 & 0,3 & 18,5 & 12,5 & 150,6 \\
\hline V1S14 & $0-20$ & 164,8 & 1,4 & 27,2 & 1,0 & 0,3 & 15,3 & 14,6 & 142,2 \\
\hline V1S15 & $0-20$ & 172,6 & 1,4 & 29,2 & 1,0 & 0,3 & 17,0 & 12,3 & 151,4 \\
\hline V1S16 & $0-20$ & 157,4 & 1,3 & 25,5 & 0,9 & 0,3 & 17,5 & 19,8 & 143,8 \\
\hline V1S17 & $0-20$ & 180,1 & 1,4 & 26,5 & 1,0 & 0,3 & 15,2 & 17,3 & 162,6 \\
\hline V1S18 & $0-20$ & 162,3 & 1,4 & 25,4 & 0,9 & 0,3 & 16,8 & 18,1 & 165,3 \\
\hline V1S19 & $0-20$ & 158,1 & 1,2 & 23,1 & 0,8 & 0,3 & 20,6 & 11,9 & 143,6 \\
\hline V1S20 & $0-20$ & 155,1 & 1,6 & 28,1 & 1,2 & 0,3 & 22,9 & 22,0 & 157,8 \\
\hline V1S21 & $0-20$ & 158,8 & 2,1 & 22,5 & 1,4 & 0,4 & 17,2 & 20,8 & 187,6 \\
\hline V1S22 & $0-20$ & 170,9 & 2,6 & 19,7 & 1,5 & 0,3 & 17,2 & 15,1 & 166,8 \\
\hline V1S23 & $0-20$ & 149,6 & 1,7 & 23,2 & 1,1 & 0,3 & 14,2 & 23,6 & 181,9 \\
\hline V1S24 & $0-20$ & 172,6 & 2,1 & 22,5 & 1,3 & 0,3 & 20,0 & 12,7 & 161,9 \\
\hline V1S24A & $0-20$ & 152,8 & 1,7 & 23,3 & 1,1 & 0,3 & 17,9 & 19,0 & 179,2 \\
\hline V1S25 & $0-20$ & 154,1 & 1,5 & 20,8 & 1,0 & 0,3 & 14,6 & 8,5 & 118,0 \\
\hline V1S26 & $0-20$ & 162,6 & 1,6 & 21,1 & 1,0 & 0,9 & 20,7 & 18,6 & 123,1 \\
\hline V1S27 & $0-20$ & 186,1 & 2,2 & 24,2 & 1,4 & 2,0 & 20,1 & 11,6 & 134,7 \\
\hline V1S28 & $0-20$ & 164,2 & 1,7 & 33,9 & 1,1 & 0,3 & 18,7 & 10,1 & 124,5 \\
\hline V1S29 & $0-20$ & 166,9 & 2,1 & 19,5 & 1,4 & 0,3 & 21,2 & 9,8 & 140,0 \\
\hline V1S30 & $0-20$ & 144,0 & 1,4 & 22,3 & 1,0 & 0,3 & 18,0 & 18,7 & 155,2 \\
\hline V1S31 & $0-20$ & 146,0 & 2,8 & 21,1 & 1,6 & 0,3 & 22,0 & 19,4 & 184,9 \\
\hline V1S32 & $0-20$ & 110,3 & 1,3 & 26,9 & 1,0 & 0,3 & 22,4 & 22,3 & 205,7 \\
\hline V1P01 & $0-20$ & 127,8 & 1,7 & 24,4 & 1,2 & 0,3 & 70,8 & 37,6 & 111,3 \\
\hline V1P02 & $20-40$ & 114,3 & 1,2 & 16,2 & 0,8 & 0,3 & 17,7 & 9,6 & 75,3 \\
\hline V1P03 & $40-60$ & 118,1 & 1,9 & 15,9 & 1,2 & 0,3 & 13,3 & 3,2 & 74,3 \\
\hline V1P04 & $60-80$ & 114,9 & 1,2 & 17,3 & 1,0 & 0,3 & 12,1 & 3,1 & 89,2 \\
\hline V1P05 & $80-100$ & 102,7 & 0,9 & 14,0 & 0,7 & 0,3 & 10,2 & 1,9 & 64,8 \\
\hline V1P06 & $100-127$ & 97,3 & 1,5 & 14,2 & 1,1 & 0,3 & 8,9 & 1,9 & 64,2 \\
\hline V1P07 & 0-17 & 133,7 & 2,0 & 22,7 & 1,3 & 0,3 & 58,8 & 33,0 & 112,1 \\
\hline V1P08 & $17-34$ & 128,2 & 2,0 & 14,9 & 1,3 & 0,3 & 25,3 & 4,8 & 87,9 \\
\hline V1P09 & $34-51$ & 135,9 & 1,7 & 18,3 & 1,2 & 0,3 & 15,2 & 1,9 & 83,0 \\
\hline V1P10 & $51-68$ & 114,7 & 1,2 & 16,2 & 0,8 & 0,3 & 12,7 & 1,9 & 70,6 \\
\hline V1P11 & $68-85$ & 114,9 & 1,7 & 17,0 & 1,1 & 0,3 & 15,5 & 1,9 & 75,6 \\
\hline V1P12 & $85-103$ & 155,6 & 1,9 & 17,5 & 1,1 & 0,3 & 12,6 & 1,9 & 100,3 \\
\hline V1P13 & $0-20$ & 148,7 & 2,8 & 27,3 & 1,6 & 4,1 & 80,1 & 26,8 & 156,8 \\
\hline V1P14 & $20-40$ & 140,2 & 2,3 & 15,8 & 1,3 & 0,3 & 37,0 & 11,7 & 111,0 \\
\hline V1P15 & $40-60$ & 159,0 & 2,4 & 16,6 & 1,3 & 0,3 & 15,4 & 4,0 & 116,0 \\
\hline V1P16 & $60-80$ & 180,8 & 2,1 & 19,6 & 1,2 & 1,5 & 12,1 & 3,5 & 108,3 \\
\hline V1P17 & $80-100$ & 149,6 & 2,1 & 15,7 & 1,3 & 0,3 & 10,2 & 10,5 & 93,1 \\
\hline V1P18 & 0-19 & 118,5 & 2,4 & 17,9 & 1,3 & 4,5 & 23,0 & 17,7 & 177,9 \\
\hline V1P19 & $19-38$ & 192,9 & 1,9 & 19,5 & 1,1 & 0,3 & 19,0 & 1,9 & 127,6 \\
\hline V1P20 & $38-57$ & 145,9 & 1,7 & 20,4 & 1,0 & 0,3 & 19,5 & 1,9 & 83,2 \\
\hline V1P21 & $57-76$ & 168,6 & 2,4 & 15,7 & 1,3 & 0,3 & 14,9 & 3,5 & 89,8 \\
\hline V1P22 & $76-95$ & 163,8 & 1,2 & 16,8 & 1,0 & 0,3 & 11,4 & 3,4 & 77,9 \\
\hline V1P23 & $95-114$ & 194,6 & 2,3 & 12,0 & 1,3 & 0,3 & 17,2 & 4,1 & 73,3 \\
\hline V1P29 & $10-30$ & 119,4 & 2,2 & 18,4 & 1,2 & 1,1 & 21,2 & 11,1 & 134,6 \\
\hline V1P28 & $40-60$ & 122,0 & 2,5 & 25,8 & 1,3 & 2,9 & 25,3 & 19,6 & 179,1 \\
\hline V1P27 & $70-90$ & 147,5 & 1,8 & 29,0 & 1,0 & 2,0 & 19,1 & 15,0 & 191,3 \\
\hline V1P26 & $100-120$ & 145,6 & 1,3 & 24,0 & 0,7 & 2,3 & 17,5 & 14,1 & 162,8 \\
\hline V1P25 & $128-148$ & 145,4 & 2,2 & 29,6 & 1,2 & 1,0 & 20,7 & 22,8 & 247 \\
\hline V1P24 & $160-180$ & 140,4 & 2,0 & 32,8 & 1,2 & 1,8 & 22,9 & 29,7 & 190,1 \\
\hline BS & $180-193$ & 67,6 & 2,3 & 12,1 & 1,3 & 0,3 & 15,9 & 5,9 & 70,2 \\
\hline V1P30 & $30-53$ & 179,8 & 2,1 & 16,3 & 1,1 & 0,3 & 15,2 & 5,8 & 130,0 \\
\hline V1P31 & $53-76$ & 74,3 & 1,9 & 21,4 & 1,3 & 0,3 & 19,9 & 25,6 & 209,9 \\
\hline V1P32 & $76-100$ & 47,3 & 0,9 & 17,5 & 0,7 & 0,3 & 13,0 & 20,5 & 196,4 \\
\hline
\end{tabular}

BS - Base Sample 
Table 3c - Heavy metal contents $(\mathrm{mg} / \mathrm{kg})$ in soil profile from Vereda Urbana. Depth in $\mathrm{cm}$.

\begin{tabular}{|c|c|c|c|c|c|c|c|c|c|}
\hline Sample & Depth $(\mathrm{cm})$ & $\mathrm{Cr}$ & Co & $\mathbf{C u}$ & Cd & $\mathbf{N i}$ & $\mathbf{Z n}$ & $\mathbf{B a}$ & $\mathbf{P b}$ \\
\hline V3P01 & $39-52$ & 76,1 & 5,9 & 46,1 & 1,0 & 12,7 & 33,7 & 183,0 & 92,2 \\
\hline V3P02 & $29-35$ & 121,2 & 6,4 & 75,2 & 0,6 & 22,1 & 47,0 & 295,3 & 128,7 \\
\hline V3P03 & $0-29$ & 205,4 & 5,7 & 87,7 & 0,8 & 21,1 & 47,9 & 291,1 & 161,8 \\
\hline V3P04 & $0-20$ & 30,1 & 2,1 & 15,9 & 0,8 & $<0,3$ & 20,1 & 40,0 & 22,3 \\
\hline V3P05 & $20-40$ & 47,4 & 5,1 & 18,8 & 1,3 & 6,2 & 28,6 & 115,1 & 56,9 \\
\hline V3P06 & $48-65$ & 47,9 & 4,0 & 30,7 & 0,9 & 10,8 & 60,7 & 141,6 & 68,1 \\
\hline V3P07 & $76-96$ & 59,7 & 2,9 & 34,8 & 0,7 & 4,6 & 27,0 & 157,5 & 64,9 \\
\hline V3S01 & $0-20$ & 38,9 & 2,2 & 21,9 & 0,7 & 2,8 & 17,7 & 42,3 & 47,9 \\
\hline V3S02 & $0-20$ & 39,3 & 2,8 & 19,8 & 0,9 & 1,1 & 30,8 & 46,8 & 60,6 \\
\hline V3S03 & $0-20$ & 59,7 & 4,9 & 25,8 & 1,0 & 10,8 & 36,6 & 91,2 & 89,4 \\
\hline V3S04 & $0-20$ & 65,3 & 5,3 & 32,6 & 0,9 & 15,6 & 40,5 & 148,8 & 103,9 \\
\hline V3S05 & $0-20$ & 48,5 & 6,1 & 31,0 & 0,8 & 14,2 & 40,6 & 228,1 & 67,1 \\
\hline V3S06 & $0-20$ & 50,6 & 3,9 & 32,9 & 0,6 & 8,5 & 33,9 & 154,6 & 66,6 \\
\hline V3S07 & $0-20$ & 53,6 & 5,4 & 34,1 & 0,7 & 15,5 & 48,4 & 134,5 & 63,9 \\
\hline V3S08 & $0-20$ & 58,5 & 5,9 & 33,6 & 1,0 & 15,3 & 44,8 & 131,6 & 71,3 \\
\hline V3S09 & $0-20$ & 60,5 & 7,5 & 35,2 & 1,1 & 18,5 & 52,5 & 179,6 & 62,6 \\
\hline V3S10 & $0-20$ & 45,6 & 3,9 & 22,2 & 1,0 & 6,9 & 21,4 & 130,6 & 48,6 \\
\hline V3S11 & $0-20$ & 61,0 & 6,2 & 26,0 & 1,0 & 15,2 & 31,6 & 183,4 & 54,3 \\
\hline V3S12 & $0-20$ & 64,5 & 4,2 & 24,6 & 0,9 & 11,4 & 34,7 & 163,7 & 51,6 \\
\hline V3S13 & $0-20$ & 54,1 & 5,1 & 32,5 & 0,7 & 14,5 & 63,4 & 158,3 & 48,0 \\
\hline V3S14 & $0-20$ & 52,0 & 5,2 & 28,5 & 0,8 & 15,0 & 35,8 & 180,4 & 60,5 \\
\hline V3S15 & $0-20$ & 62,1 & 6,5 & 34,4 & 0,9 & 17,5 & 42,9 & 207,2 & 59,7 \\
\hline V3S16 & $0-20$ & 23,4 & 1,6 & 15,7 & 0,7 & $<0,3$ & 15,4 & 25,7 & 29,1 \\
\hline V3S17 & $0-20$ & 33,4 & 2,2 & 19,9 & 0,7 & 1,5 & 21,9 & 48,4 & 47,9 \\
\hline V3S18 & $0-20$ & 48,6 & 3,3 & 28,6 & 0,8 & 9,6 & 24,6 & 77,7 & 75,0 \\
\hline V3S19 & $0-20$ & 49,9 & 4,0 & 28,7 & 1,0 & 12,6 & 28,1 & 75,6 & 91,2 \\
\hline V3S20 & $0-20$ & 69,0 & 6,3 & 32,9 & 1,4 & 13,4 & 45,7 & 85,5 & 113,0 \\
\hline V3S21 & $0-20$ & 56,4 & 5,3 & 32,1 & 1,0 & 15,8 & 30,3 & 100,2 & 93,4 \\
\hline V3S22 & $0-20$ & 46,4 & 4,7 & 27,2 & 0,9 & 15,7 & 25,2 & 79,6 & 74,8 \\
\hline V3S23 & $0-20$ & 45,4 & 4,0 & 24,4 & 0,8 & 12,3 & 23,7 & 73,9 & 123,5 \\
\hline V3S24 & $0-20$ & 58,7 & 4,5 & 34,3 & 0,9 & 13,6 & 27,6 & 85,8 & 95,9 \\
\hline V3S25 & $0-20$ & 60,8 & 4,9 & 37,3 & 0,9 & 16,9 & 31,4 & 101,4 & 103,8 \\
\hline V3S26 & $0-20$ & 42,5 & 3,7 & 26,3 & 0,9 & 9,9 & 27,2 & 71,5 & 73,9 \\
\hline V3S27 & $0-20$ & 45,6 & 4,5 & 28,5 & 0,9 & 13,8 & 27,3 & 85,2 & 93,0 \\
\hline V3S28 & $0-20$ & 49,7 & 6,0 & 31,8 & 1,1 & 19,7 & 36,0 & 92,5 & 94,4 \\
\hline V3S29 & $0-20$ & 46,7 & 4,8 & 25,3 & 1,1 & 12,0 & 26,2 & 94,2 & 81,1 \\
\hline V3S30 & $0-20$ & 42,6 & 4,1 & 23,9 & 0,8 & 14,6 & 30,3 & 88,6 & 68,6 \\
\hline
\end{tabular}

\section{RESULTS AND DISCUSSION}

The obtained soil profile characteristics were compared with the GPR-results to decipher the correlation of these two methods

\subsection{GPR-RESULTS}

In each of the studied veredas, three geophysical profiles were performed. Always two of these profiles intersect at one point and have approximately perpendicular directions to each another. The data were acquired with 100 $\mathrm{MHz}$ antennas in the common-off-set mode. and to see the importance of previous GPR survey to support chemical, mineralogical and genetic investigations.

The third profile was in CMP mode, to obtain the velocity of the EM wave on the subsurface. The photo from the Vereda Laçador (Figure 6) shows the opening of the profile trails, the proper acquisition of the profiles, as well as the relative position between them. 


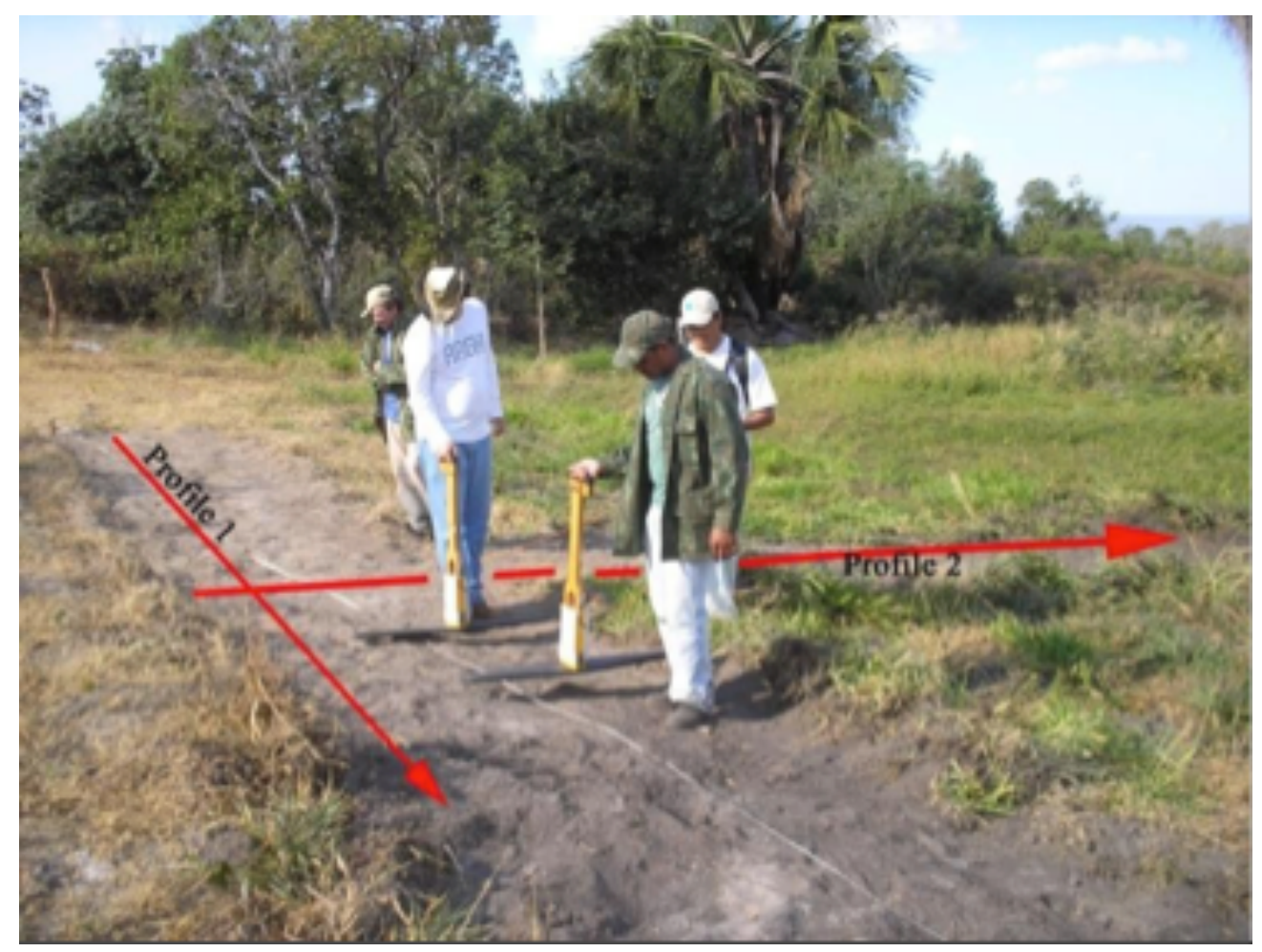

Figure 6

Photos of the GPR profile acquisition at the Vereda Laçador.

In the Radargram from profile 1 obtained at Vereda Laçador (Figure 7), the reflection of the EM wave is related to the low depth groundwater level of the area. This level was determined by the observations obtained in the drill holes near the site of acquisition of the
GPR profiles. This reflection is marked blue in the interpreted profile. The presence of prominent but discontinuous reflections, indicate the presence of alluvial sediments and their internal structures.
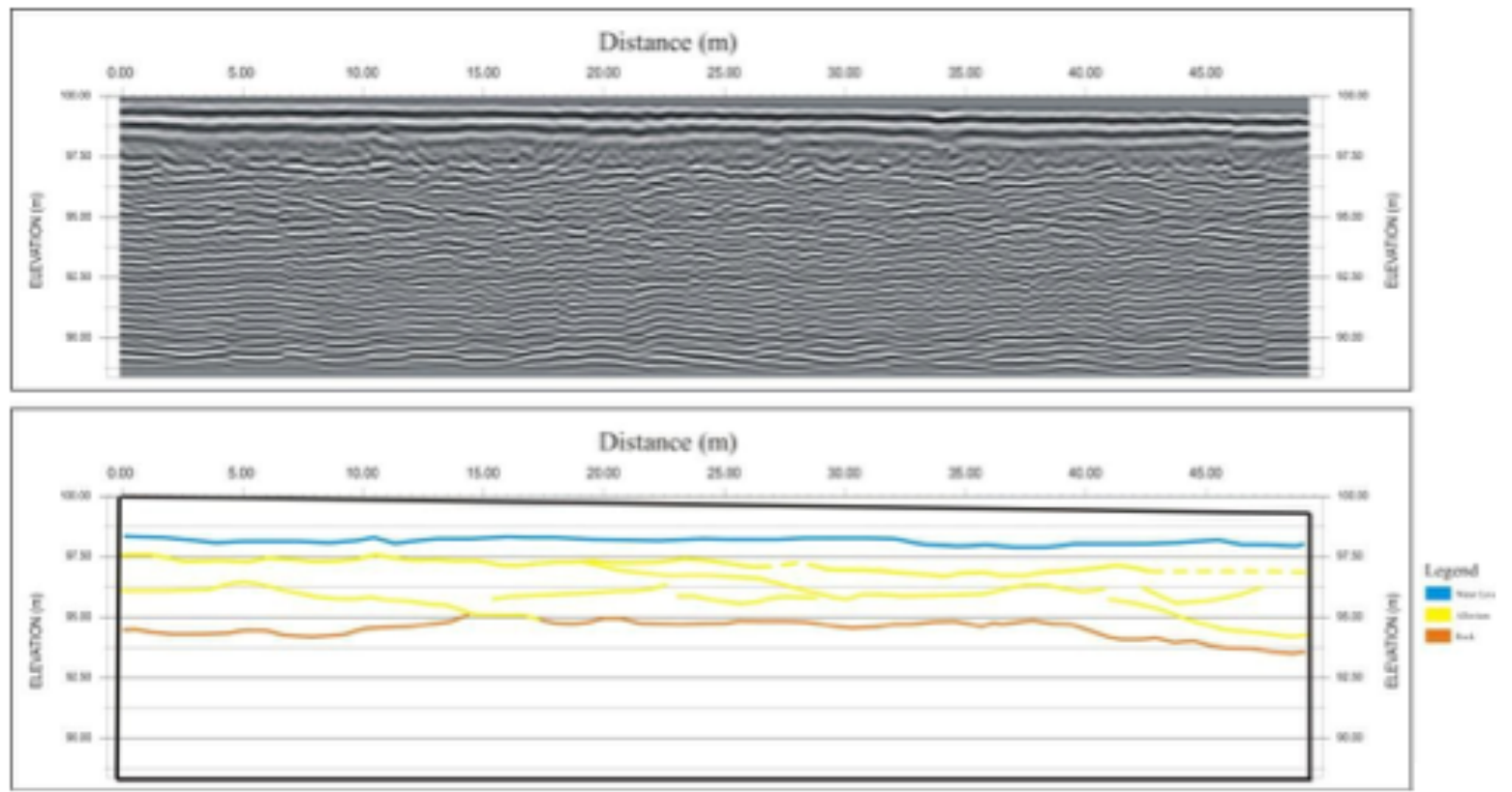

Figure 7

Radargram 1 from Vereda Laçador and its interpretation. 
The Radargram of Profile 2 in this Vereda (Figure 8) presents similar patterns like the Radargram 1 (Figure 7). The reflections of the groundwater level are indicated in blue, the discontinuities and overlapped reflections are marked in yellow presenting the pattern of cross stratification, typical of fluvial sedimentary deposits. This part of the
Vereda can be considered as an abandoned river arm, with its channel and margins. Below this upper level, the reflections of the basement with completely different patterns from the one that occurs in the interfaces of the alluvial situation can be observed. Also in Vereda Jaraguá the GPR-profiles were obtained perpendicularly to each another (Figure 9).
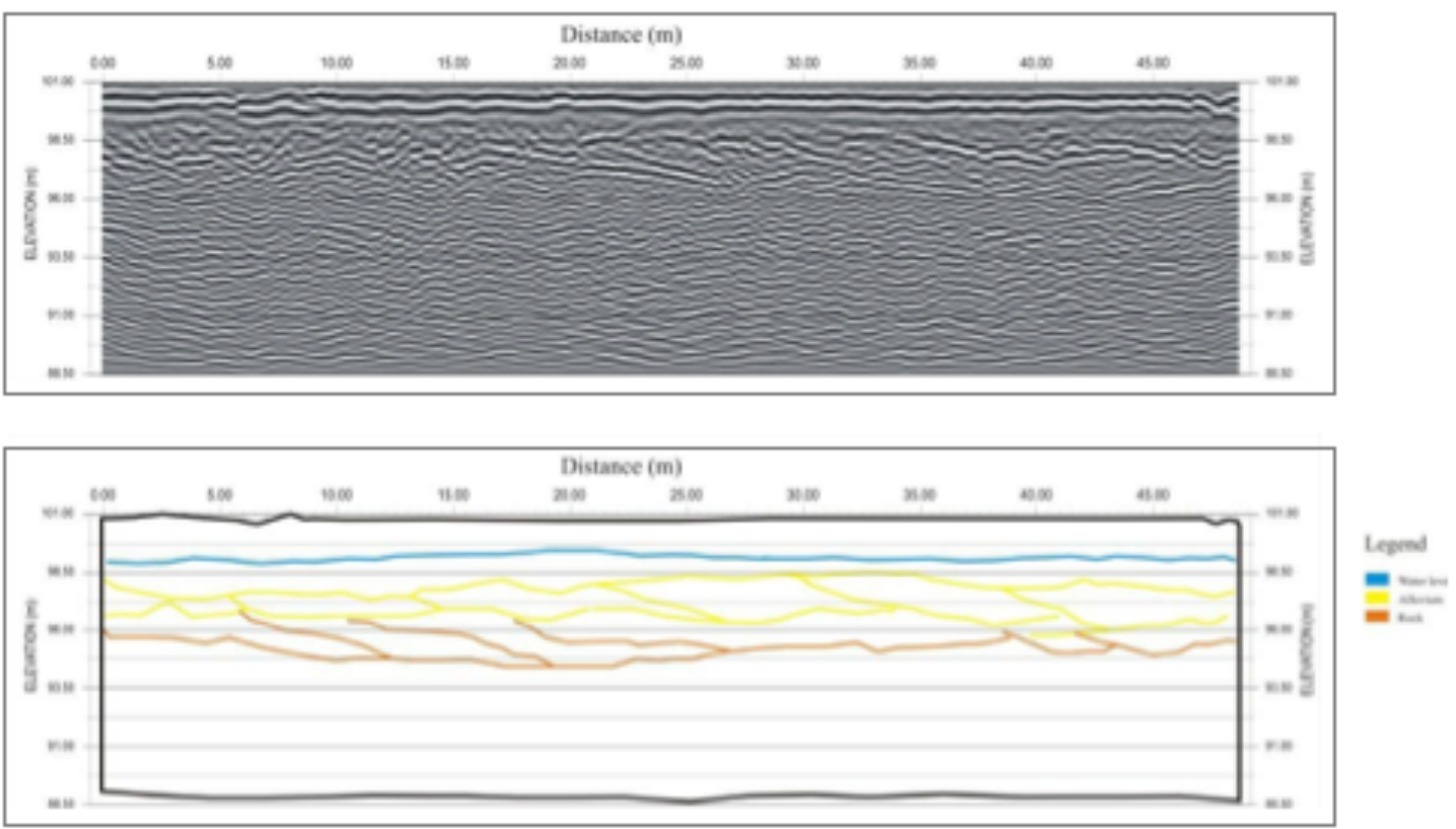

Figure 8

Second radargram of Vereda Laçador and its interpretation
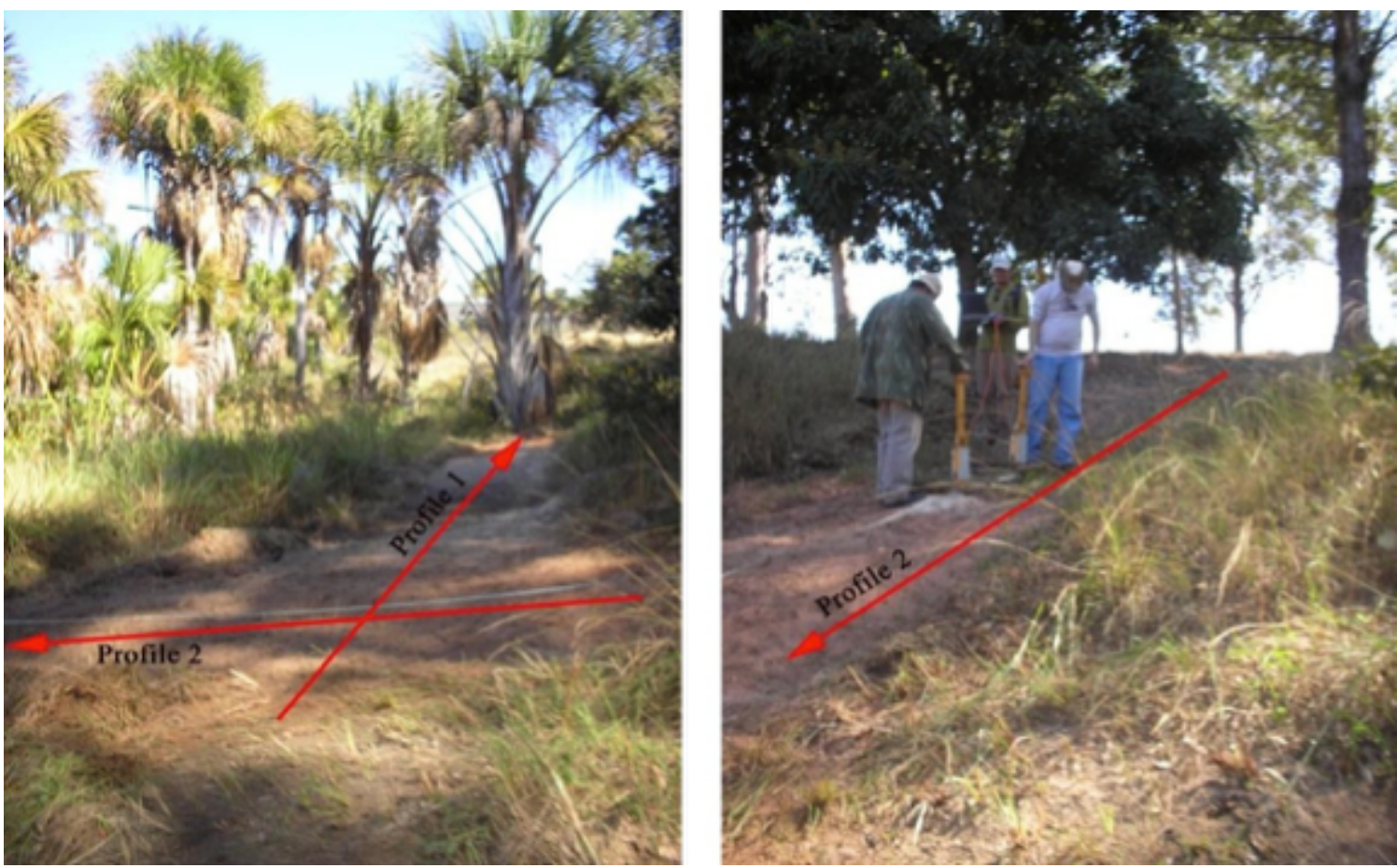

Figure 9

Photos of the locations where profiles 1 and 2 of Vereda Jaraguá were prepared and executed. 
The Radargram 1 obtained along a profile of the Vereda Jaraguá (Figure 10) shows some very distinct reflections patterns of the EM wave. Between the distances from 0 to $7 \mathrm{~m}$, one can observe the presence of a well-marked depression representing presence of a welldefined fluvial paleochannel. About $20 \mathrm{~m}$ distance another small current drainage channel can be seen, and just below them a well-defined paleochannel, however narrower than the paleochannel of the left part of the radargram. Just below the depth of $96.7 \mathrm{~m}$ in the first meters of the radargram, in the upper part the presence of a reflector with strong amplitude marking the contact between the alluvium and the saprolite can be seen. Just below this reflector, the drill holes show the presence of the not altered bedrock
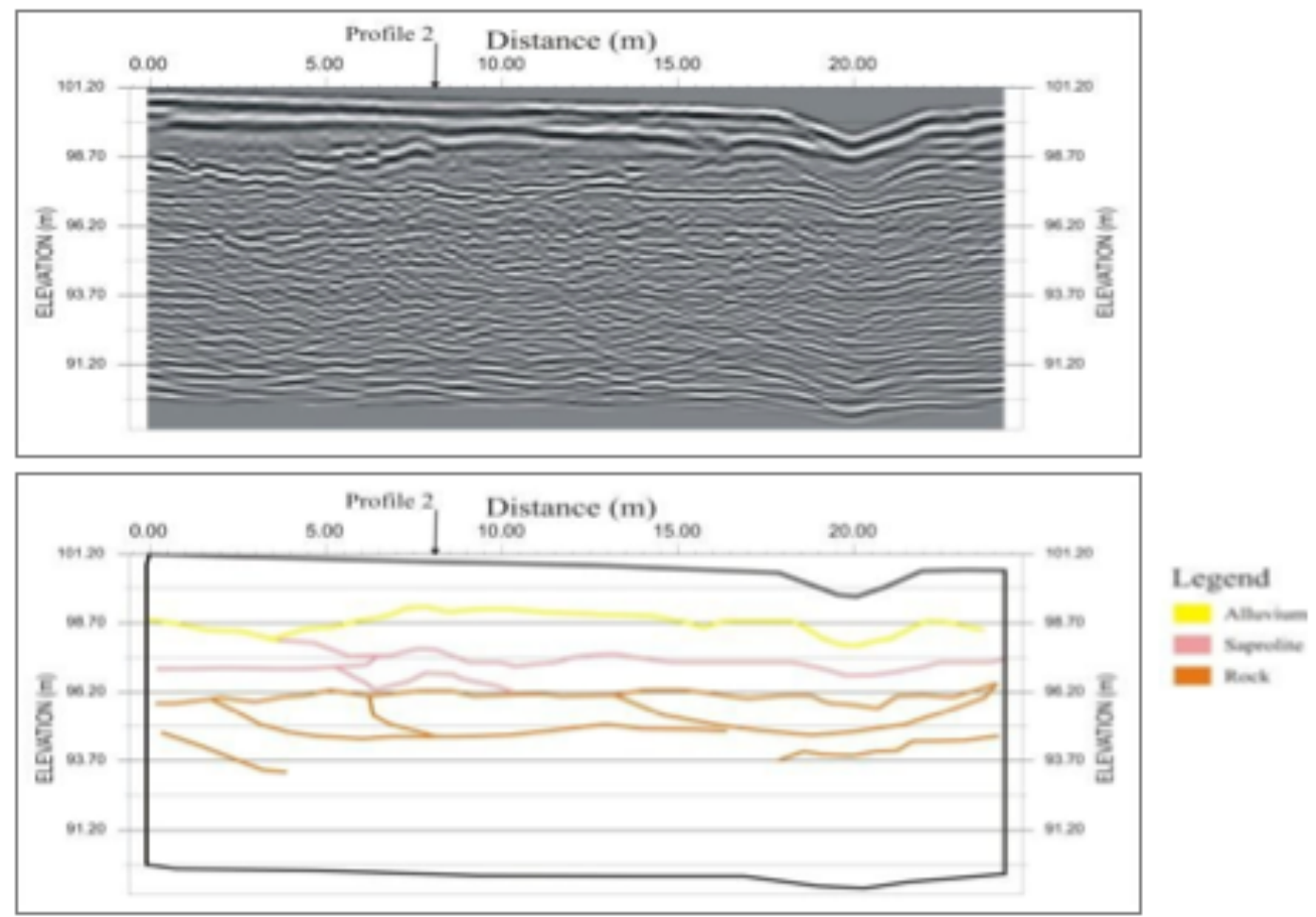

Figure 10

First radargram of the Vereda Jaraguá and its interpretation.

In the second radargram obtained in the Vereda Jaraguá, throughout the left portion of the radargram (Figure 11), a predominance of sub-horizontal-flat parallel reflectors is noted. These extend up to 15 meters away, with angular orientation in relation to the surface, indicating a newer deposit. Approaching to the center of the profile, at 5 meters depths, a truncation between different lithological strata is observed. The reflectors exhibit features with asymmetric and discontinuous pattern, possibly indicating a cross-stratified deposition. Right below in the diagram, the reflectors patterns of acrolith can be observed. At the intersection between the profiles, the observations are congruent for the two profiles

In the area of Vereda Urbana two profiles were obtained, one along a road and another cutting the Vereda perpendicularly (Figure 12). In the upper part of the radargram obtained in Profile 1 (Figure 13), a wavy, well-highlighted reflector is observed. This structure is related to the presence to small fluvial paleochannels, and some internal reflectors indicating the sedimentation process of the alluvial sediments. Below it is a more flat-plane reflector indicating the presence of the paleosurface, formed by the rocky saprolitic basement.

The presence of sub vertical planes, related to discontinuities or fractures present in the rock is noted. In the radargram of profile 2 (Figure 14), perpendicular to the first, in the upper part the reflections on the alluvial sediments can be observed. Just below, the presence of a continuous and flat reflector indicates the contact to the rocky basement. In this basement formed by sandstone, flat reflectors are observed, showing possible internal sedimentation plans during its deposition. 

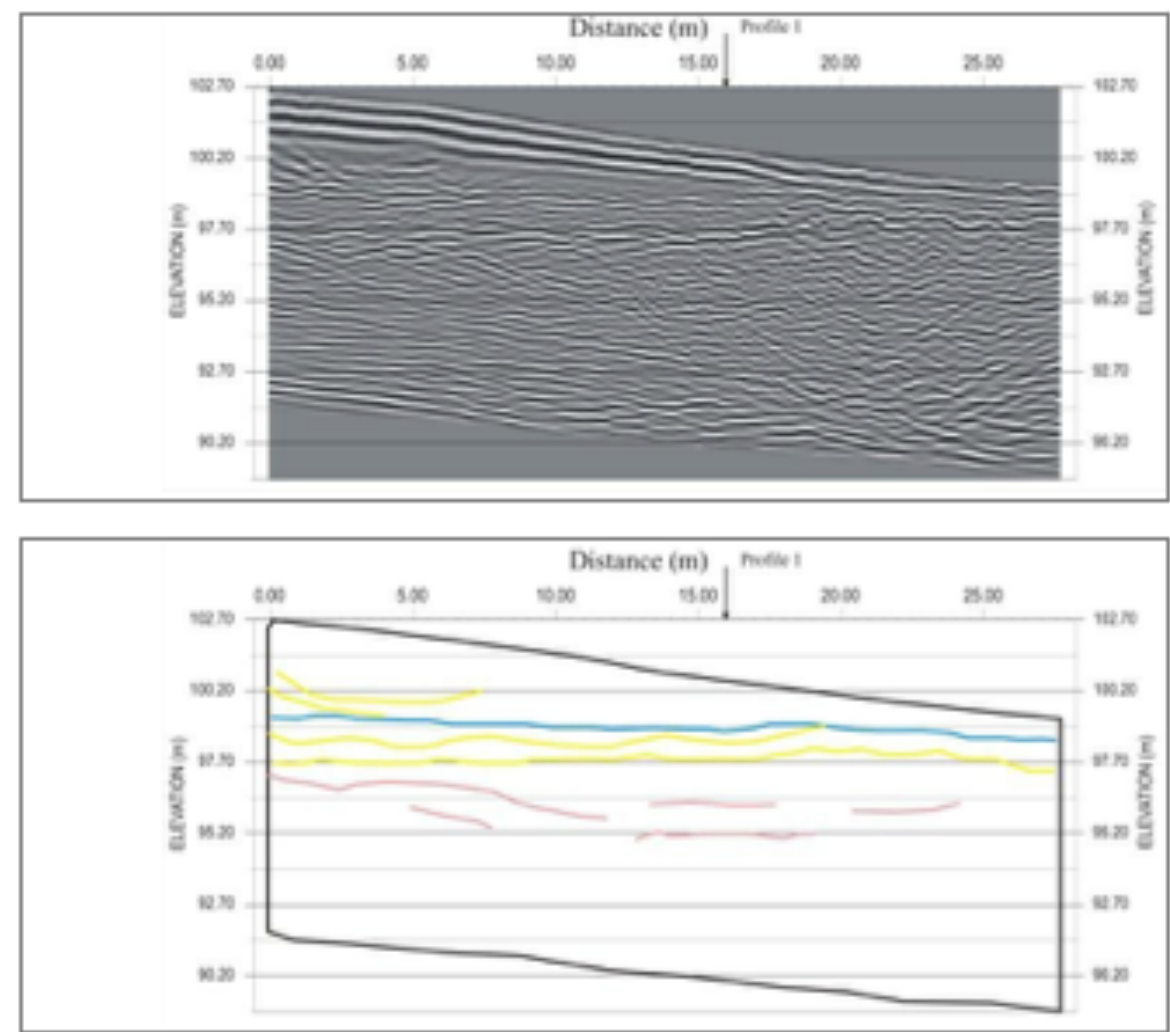

Legend

E. Wuar Levi

IVInim

Simler

Figure 11

Second radargram of the Vereda Jaraguá and its interpretation.
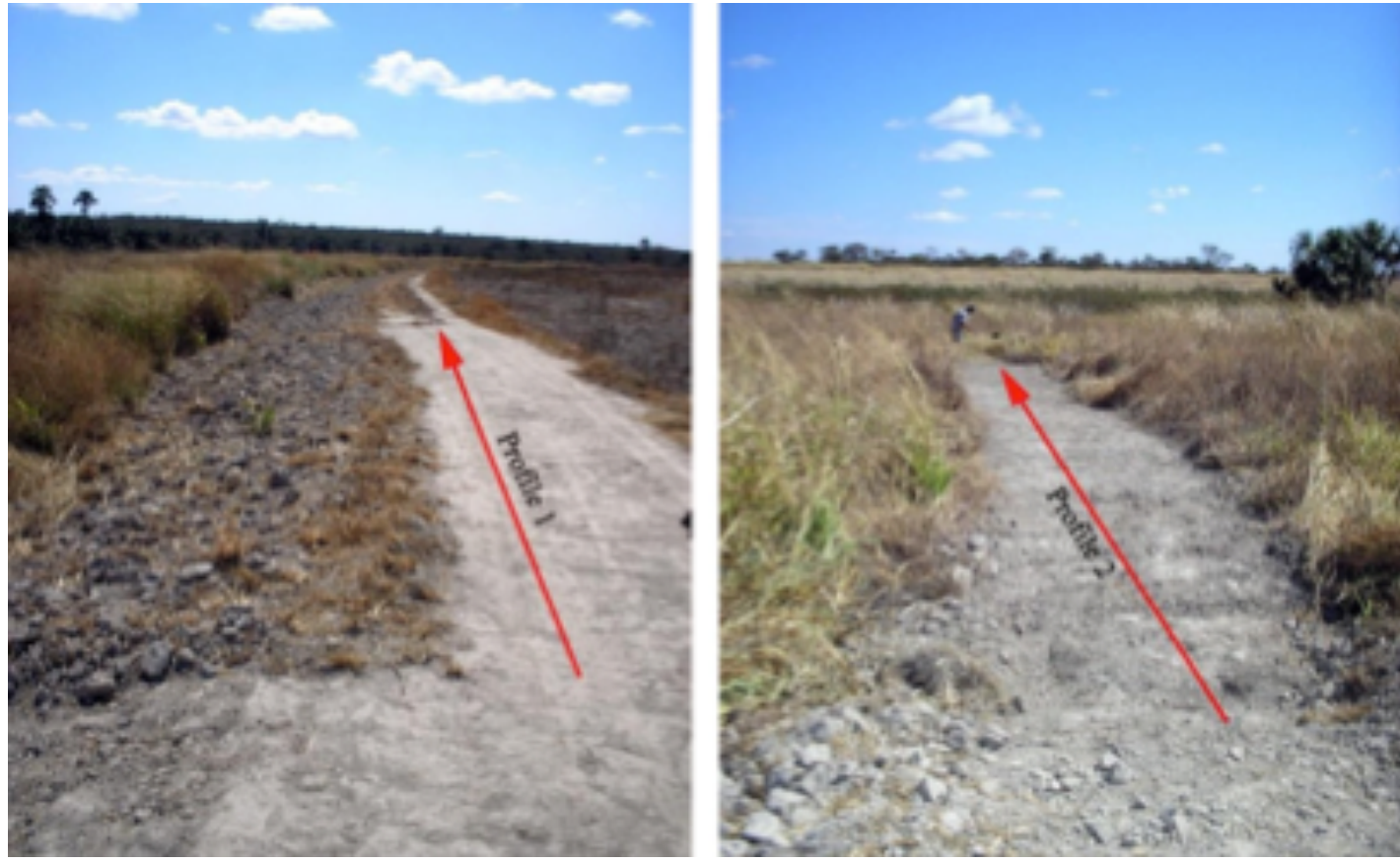

Figure 12

Photographs of Vereda Urbana with the localization of the GPR profile lines. 

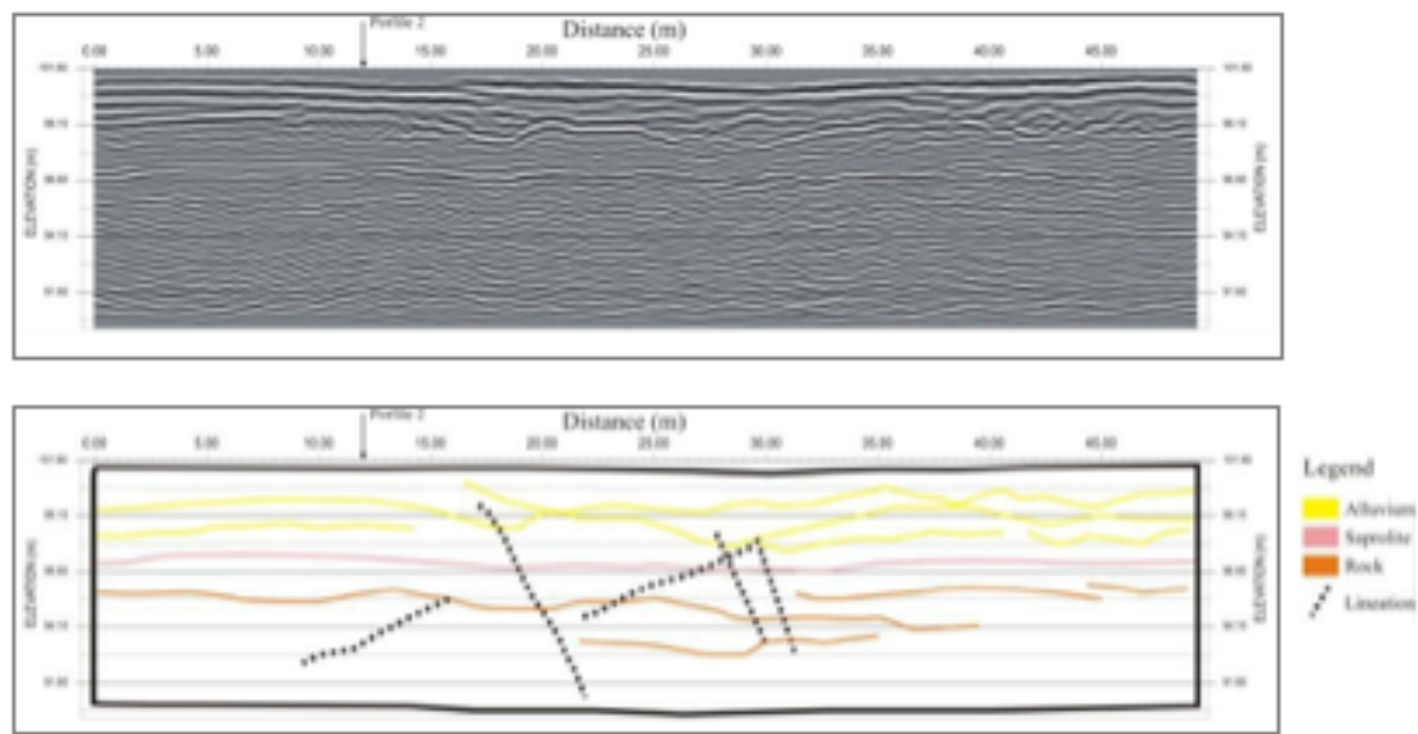

Figure 13

First radargram of Vereda Urbana and its interpretation.
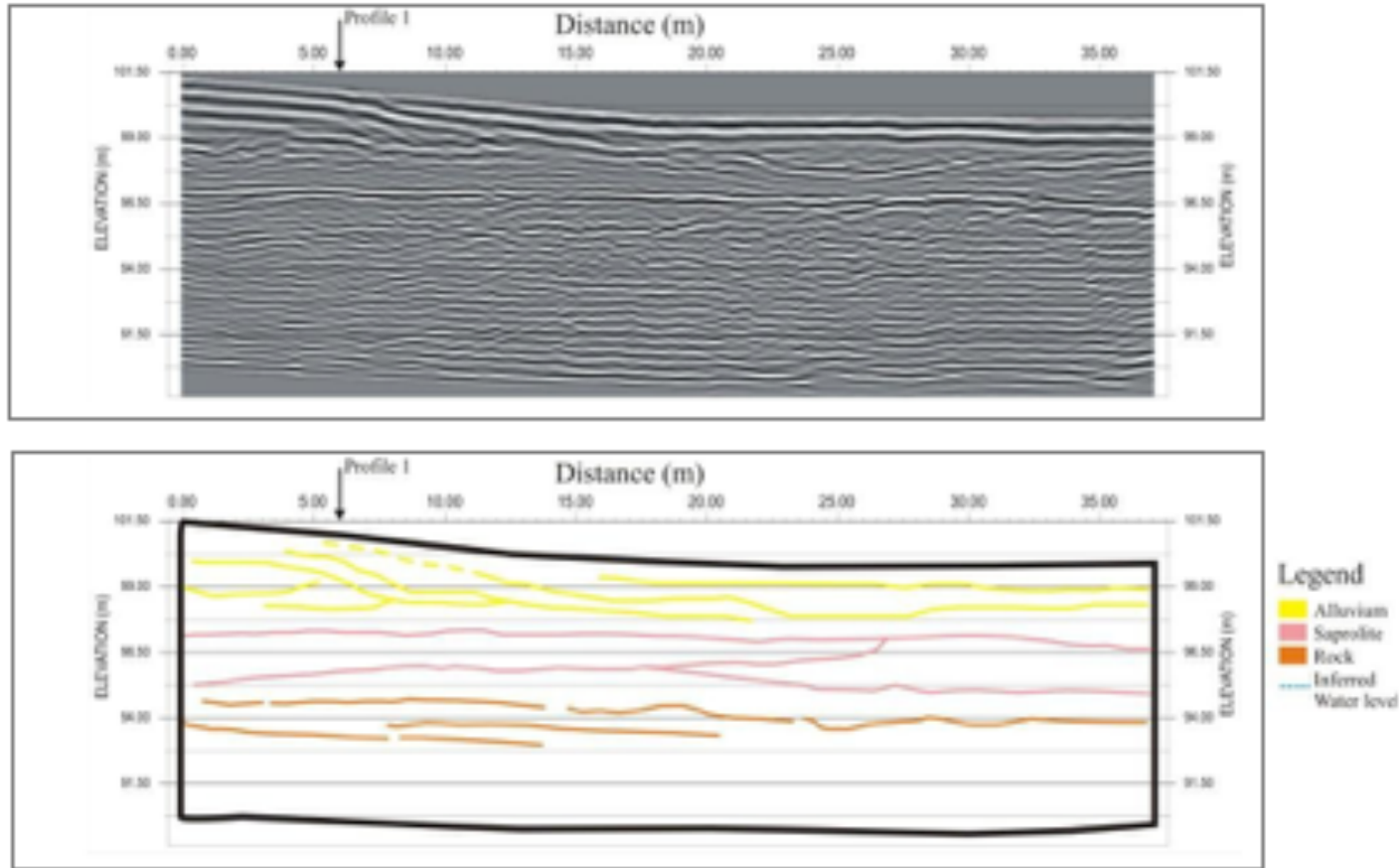

Figure 14 Second radargram of Vereda Urbana and its interpretation

\subsection{SOIL PROFILE INTERPRETATION}

Using the obtained information, the results of GPR, soil profiles, mineralogy and grain

size distribution were combined to create additive profiles over the selected veredas.

\subsubsection{SOIL COLOR}

In the Vereda Laçador samples, the color ranges from $5 \mathrm{Y}$ (light grey) to $2.5 \mathrm{~N}$ (black), in Vereda Jaraguá from 2.5 Y (light samples of Vereda Urbano the colors are distributed from 10 YR 7/3 (brown with very 
slightly clear-greyed touch) to 10 YR 2/1 (black). The color distribution is typical for

\subsubsection{MINERAL COMPOSITION}

The obtained results by X-rays diffraction indicate a predominance of quartz, kaolinite, gibbsite and subordinate of muscovite as important constituents of mineralogy of the soil

\subsubsection{SOIL CHEMISTRY}

As shown in tables 1, 2 and $3(a, b$ and $c)$ it is possible to see that the results are connected to each other. Higher CTC indicates higher concentrations of clay minerals (Figure 15). The CTC-capacity is also correlated with the very poorly drained soils with high organic content and formed under reduced conditions.

samples. Using this data and field observations it was possible to show additive typical profiles for the three investigated veredas.

mineral distribution in the profile samples. The GPR defined contacts are indicative for the changes in profile mineral composition (grainsize; composition) and therefore in this way for the metal concentrations.
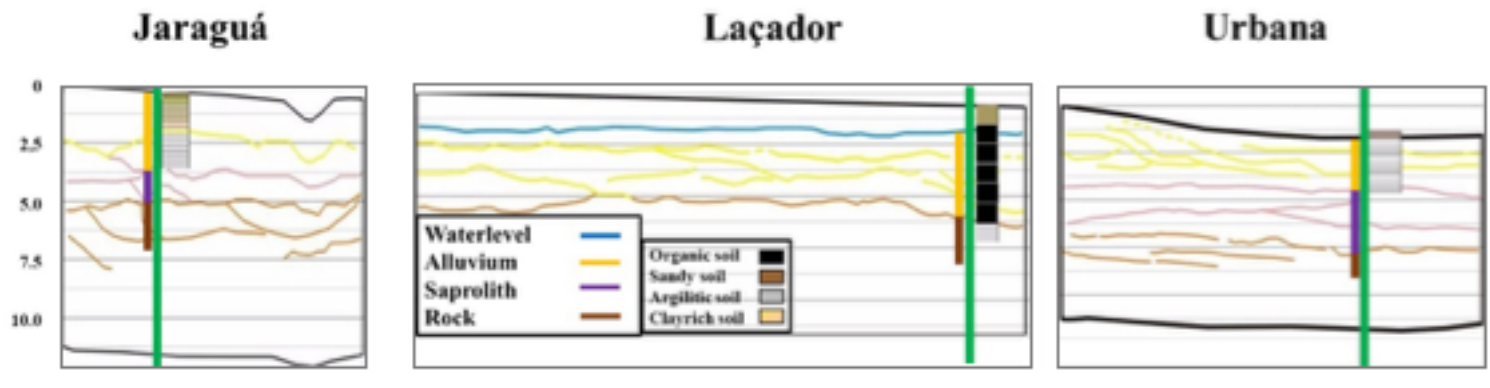

Figure 15 Integration of GPR data, drill and soil sampling results. Depth approximately in meters. Green: Drill hole; left column: Rock distribution in the drill; right column: Soil profile.

\section{CONCLUSIONS}

Data from the interpretation of radargrams allow to define the structures of the subsurface of the area. These data indicate the presence of paleochannels, and favorable conditions for the slow percolation of water in the basement. Only in the profiles from Vereda Urbana the presence of lineaments in the rock can be noted. However, these lineaments did not prevent a greater accumulation of water over the basement. The structures observed in the subsurface of each of the veredas, reflectors with horizontal tendency, few fractures in the not weathered rock, conditioned the process of soil evolution present in the area with its

\section{ACKNOWLEDGMENTS}

To the UFMG, UNIMONTES and the project "Evolution of the paleo-geoQuaternary environment of the veredas of the São Francisco River basin $\mathrm{CNPq}$ (MCT/CNPq 15/2007- Universal), characteristics observed in the samples collected and analyzed.

These conditions favor a similar development, although the veredas are located in different geographical and geological positions in the basin, and even in different elevations. The GPR proved to be very efficient in observing the structures of the subsurface, revealing forms and continuities of sedimentation plans, as well as the presence of few lineaments in the sound rock and to correlate with other analytical data to form an integrated model. 


\section{REFERENCES}

ABNT - Associação Brasileira de Normas Técnicas. NBR 7181. Solo-Análise granulométrica. Rio de Janeiro. 13 p. 1984

BAGGIO, H. Contribuições naturais $e$ antropogênicas para a concentração $e$ distribuição de metais pesados em água superficial e sedimento de corrente na Bacia do Rio do Formoso, município de Buritizeiro, $M G$. Tese de Doutorado, Instituto de Geociências, Universidade Federal de Minas Gerais, 249 p. 2008

CHIAVEGATTO, J. R. S. Análise estratigráfica das seqüencias tempestística da Formação Três Marias (Proterozóico Superior), na porção meridional da Bacia do São Francisco. Dissertação de Mestrado, Departamento de Geologia, Universidade Federal de Ouro Preto, 216 p. 1992

EMBRAPA. Manual de Métodos de Análise de Solo. Empresa Brasileira de Pesquisa Agropecuária. Centro Nacional de Pesquisas de Solos. Rio de Janeiro, 2 ed. 212 p. 1997

MELO, D. R. As Veredas nos planaltos do noroeste mineiro: caracterizações pedológicas $e$ os aspectos morfológicos e evolutivos. Dissertação de Mestrado. Instituto de Geociências e Ciências Exatas, Universidade Estadual Paulista, 218 p. 1992
SGARBI, G. N. C. Arenitos Eólicos da Formação Areado (Bacia Cretácea do São Francisco): Caracterização diagênese e aspectos. Revista Brasileira de Geociências. 21(4): 342-354, 1991

SGARBI, G. N. C. Bacia Sanfranciscana: o registro do Fanerozóico da bacia do São Francisco. In: Pinto, C. P.; Martins-Neto, M. (Eds) Bacia do São Francisco: Geologia e Recursos Naturais. SBG-MG. 93-138 p. 2001

US-EPA - United States Environmetal Protection Agency. Preparation of Soil Sampling Protocols: Sampling Techniques and Strategies. United States Environmental Agency. Washington; EPA/600/R-92/128; 169p. 1992

US-EPA - United States Environmetal Protection Agency. Microwave assisted acid digestion of sediments, sludges, soils and oils. United States Environmental Agency. Washington; 30p. 2007

VIANA, V. M. F. C. Estudo Hidrogeoquímico das Veredas do Rio do Formoso no Município de Buritizeiros, Minas Gerais. Dissertação de Mestrado, Instituto de Geociências, Universidade Federal de Minas Gerais, 107 p. 2006 